Signals and Systems Group: NUIM/SS/2001/04

A new methodology for the stability analysis of pairwise triangularisable and related switching systems

Robert N. Shorten and Fiacre Ó Cairbre 


\title{
A new methodology for the stability analysis of pairwise triangularisable and related switching systems
}

\author{
Fiacre Ó Cairbre \\ Department of Mathematics \\ National University of Ireland \\ Maynooth, Co. Kildare \\ Ireland
}

\author{
Robert Shorten \\ Department of Computer Science \\ National University of Ireland \\ Maynooth, Co. Kildare \\ Ireland
}

\begin{abstract}
A sufficient condition for the existence of a Lyapunov function of the form $V(x)=$ $x^{T} P x, P=P^{T}>0, P \in \mathbb{R}^{n \times n}$, for the stable linear time invariant systems $\dot{x}=$ $A_{i} x, A_{i} \in \mathbb{R}^{n \times n}, A_{i} \in \mathcal{A}=\left\{A_{1}, \ldots, A_{m}\right\}$, is that the matrices $A_{i}$ are Hurwitz, and that a non-singular matrix $T$ exists, such that $T A_{i} T^{-1}, i \in\{1, \ldots, m\}$, is upper triangular (Mori, Mori \& Kuroe 1996, Mori, Mori \& Kuroe 1997, Liberzon, Hespanha \& Morse 1998, Shorten \& Narendra 1998). The existence of such a function, referred to as a common quadratic Lyapunov function (CQLF), is sufficient to guarantee the exponential stability of the switching system $\dot{x}=A(t) x, A(t) \in \mathcal{A}$. In this paper we investigate the stability properties of related classes of switching systems. We consider sets of matrices $\mathcal{A}$, where no single matrix $T$ exists that simultaneously transforms each $A_{i} \in \mathcal{A}$ to upper triangular form, but where a set of non-singular matrices $T_{i j}$ exist such that the matrices $\left\{T_{i j} A_{i} T_{i j}^{-1}, T_{i j} A_{j} T_{i j}^{-1}\right\}, i, j \in\{1, \ldots, m\}$, are upper triangular. We show that in general, this condition does not imply the existence of a common quadratic Lyapunov function (CQLF). Further, we also show by means of a simple example, that the condition of pairwise triangularisability is not sufficient to guarantee stability of an associated switching system. However, we show that, for special classes of related systems, the origin of the switching system. $\dot{x}=A(t) x, A(t) \in \mathcal{A}$, is globally attractive. A novel technique, referred to in this paper as state-space-embedding, is developed to derive this result. State-space-embedding is based upon the observation that the stability properties of an $n$-dimensional switching system may, on occasion,
\end{abstract}


be analysed by embedding the $n$-dimensional system in a higher dimensional system. The efficacy of this technique is demonstrated by showing the stability of two distinct classes of switching systems, and by utilising these results to design a control system for a real industrial application; namely the design of a stable automobile speed control system. 


\section{Introductory remarks}

We consider switching systems of the following form,

$$
\dot{x}=A(t) x, A(t) \in \mathcal{A}=\left\{A_{1}, A_{2}, \ldots, A_{m}\right\},
$$

where the $A_{i}, i \in\{1,2, \ldots m\}$, are constant matrices in $\mathbb{R}^{n \times n}$. The matrices $A_{i}, i \in$ $\{1,2, \ldots m\}$, are assumed to be Hurwitz (the eigenvalues of each $A_{i}$ matrix lies in the open left half of the complex plane). A sufficient condition for the exponential stability of Equation (1), and for the existence of a common quadratic Lyapunov function (CQLF), $V(x)=x^{T} P x, P=P^{T}>0, P \in \mathbb{R}^{n \times n}$, for the stable linear time invariant (LTI) systems

$$
\Sigma_{A_{i}}: \dot{x}=A_{i} x, x \in \mathbb{R}^{n}, A_{i} \in \mathcal{A}, A_{i} \in \mathbb{R}^{n \times n},
$$

is that the matrices $A_{i}$ are Hurwitz, and that a non-singular transformation $T$ exists such that $T A_{i} T^{-1}$ is upper triangular for all $i \in\{1, \ldots, m\}$. This result was first derived by Mori et al. (1997), and further discussed by Liberzon et al. (1998) and Shorten \& Narendra (1998). Unfortunately, from a practical viewpoint, the requirement of simultaneous triangularisability imposes unrealistic conditions on the matrices in the set $\mathcal{A}$. It is therefore of interest to extend the results derived by Mori et al. (1997) with a view to relaxing this requirement. In this context several authors have recently published new conditions which also guarantee exponential stability of the switching system. Typically, the approach adopted is to bound the maximum allowable perturbations of the matrix parameters from a nominal (triangularisable) set of matrices, thereby guaranteeing the existence of a CQLF; see Mori et al. (1997). In this paper we consider classes of switching systems that are closely related to those studied by Mori et al. (1997). However, rather than assuming maximum allowable perturbations from nominal matrix parameters, we explicitly assume that no single nonsingular transformation $T$ exists that simultaneously triangularises all of the matrices in $\mathcal{A}$. Rather, we assume that a number of non-singular matrices $T_{i j}$ exist, such that for each pair

of matrices in $\mathcal{A},\left\{A_{i}, A_{j}\right\}$, the matrices $\left\{T_{i j} A_{i} T_{i j}^{-1}, T_{i j} A_{j} T_{i j}^{-1}\right\}$ are upper triangular. We refer to switching systems that are constructed in this manner as pairwise triangularisable 
switching systems.

In this paper we establish a number of results concerning pairwise triangularisable switching systems. We show that while the condition of pairwise triangularisability is not sufficient to guarantee asymptotic stability, global attractivity of the origin can be established using state-space-embedding by making other assumptions. These results are important for a number of reasons. Primarily, they conclusively invalidate the conjecture made in (Shorten \& Ó Cairbre n.d.) that the condition of pairwise triangularisability alone is sufficient to guarantee asymptotic stability of (1) for arbitrary switching sequences. Secondly, we show that state-space-embedding can be utilised to prove global attractivity of the origin for several classes of switching system. This technique does not utilise concepts from quadratic Lyapunov theory and may therefore be used in situations where such functions do not exist. Asymptotic stability of the system follows from known results in the literature. Finally, these results are used to design a stable switching controller for a car speed control system.

This paper is organised as follows. Preliminary definitions and mathematical results are presented in Section 2. General pairwise triangularisable systems are discussed in Section 3. The method of state-space-embedding is formally introduced in Section 4, and the technique is applied to prove the global attractivity of a class of switching systems related to those discussed in Section 3. These results are applied to the design of a stable speed control system for an automobile in Section 5 . 


\section{Preliminary results}

In this section we introduce some simple concepts and definitions (from Narendra \& Annaswamy (1989)) which are useful in the remainder of the paper.

(i) The switching system : Consider the linear time-varying system

$$
\dot{x}=A(t) x,
$$

where $x \in \mathbb{R}^{n}$, and where the matrix switches between the matrices $A_{i} \in \mathbb{R}^{n \times n}$ belonging to the set $\mathcal{A}=\left\{A_{1}, \ldots, A_{m}\right\}$. We shall refer to this as the switching system. The time-invariant linear system $\dot{x}=A_{i} x$, denoted $\Sigma_{A_{i}}$ is referred to as the $i^{\text {th }}$ constituent system.

Suppose that (1) is described by the $\gamma^{t h}$ system $\dot{x}=A_{\gamma} x$ over a time interval $\left[t_{\gamma}, t_{\gamma+1}\right]$. By definition, the next system that we switch to, say the $(\gamma+1)^{\text {th }}$ system, starts at time $t_{\gamma+1}$ with initial conditions equal to the terminal conditions of the $\gamma^{\text {th }}$ system at time $t_{\gamma+1}$.

(ii) Stability of the origin : The equilibrium state $x=0$ of Equation (3) is said to be stable if for every $\epsilon>0$ and $t_{0} \geq 0$, there exists a $\delta\left(\epsilon, t_{0}\right)>0$ such that $\left\|x_{0}\right\|<\delta\left(\epsilon, t_{0}\right)$ implies that $\left\|x\left(t ; x_{0}, t_{0}\right)\right\|<\epsilon, \forall t \geq t_{0}$.

(iii) Attractivity of the origin : The equilibrium state $x=0$ of Equation (3) is said to be attractive if for some $\rho>0$, and for every $\theta>0$ and $t_{0}$, there exists a number $T\left(\theta, x_{0}, t_{0}\right)$ such that $\left\|x_{0}\right\|<\rho$ implies that $\left\|x\left(t ; x_{0}, t_{0}\right)\right\|<\theta, \forall t \geq t_{0}+T$.

(iv) Global attractivity of the origin : The equilibrium state $x=0$ of Equation (3) is said to be globally attractive if $\lim _{t \rightarrow \infty} x\left(t ; x_{0}, t_{0}\right)=0$, for all initial conditions $x_{0}$ and for all $t_{0} \geq 0$. 
(v) Asymptotic stability : The equilibrium state of Equation (3) is said to be asymptotically stable if it is both stable and attractive.

(vi) Common quadratic Lyapunov function: In the following discussion we refer to common quadratic Lyapunov functions (CQLF's). A common quadratic Lyapunov function is defined as follows.

Consider the switching system defined in (3) where all the elements of $\mathcal{A}$ are Hurwitz. The quadratic function

$$
V(x)=x^{T} P x, \quad P=P^{T}>0, \quad P \in \mathbb{R}^{n \times n},
$$

is said to be a common quadratic Lyapunov function for each of the constituent subsystems $\Sigma_{A_{i}}, i \in\{1, \ldots, m\}$, if symmetric positive definite matrices $Q_{i}, i \in\{1, \ldots, m\}$, exist such that the matrix $P$ is a solution of the matrix equations

$$
A_{i}^{T} P+P A_{i}=-Q_{i}
$$

The existence of a common quadratic Lyapunov function implies the exponential stability of the switching system (3) as discussed by Narendra \& Balakrishnan (1994).

We note the following important result.

Theorem 2.1 : (Shorten \& Narendra (1997),Shorten, Ó Cairbre \& Curran (2000)) A sufficient condition for the existence of a switching sequence, such that the system (1) is unstable, is that there exist non-negative constants $\left\{\alpha_{1}, \alpha_{2}, \ldots, \alpha_{M}\right\}$, such that the matrix pencil $\sum_{i=1}^{M} \alpha_{i} A_{i}, \alpha_{i} \geq 0, \sum_{i=1}^{M} \alpha_{i}>0$ has an eigenvalue with a positive real part.

Proof : Appendix. 


\section{Pairwise triangularizable systems}

As discussed in the introduction, switching systems that are constructed from constituent dynamic systems whose system matrices are simultaneously triangularisable have been the subject of much interest in the switching system community. The exponential stability of systems in this form can be readily established using standard arguments (an analytical expression for their solution is readily obtained). Nevertheless, the widespread interest in these systems is motivated by the fact that the condition of simultaneous triangularisability is one of the few known (simple) conditions that guarantees the existence of a CQLF for each of the constituent systems. While the theoretical interest in these systems is justified, practical applications motivate a number of open questions pertaining to related classes of switching systems. In this Section we consider switching systems where any two matrices in the switching set $\mathcal{A}$ are Hurwitz, and can be simultaneously triangularised. We refer to systems in this form as pairwise triangularisable switching systems. In this context we note the following important questions.

(i) Is the condition of pairwise triangularisability sufficient to guarantee the existence of a CQLF for each of the constituent sub-systems?

(ii) Is the condition of pairwise triangularisability sufficient to guarantee asymptotic stability of the origin of (1)?

(iii) In the event of negative answers to (i) and (ii) (which are not equivalent), is it possible to specify extra conditions in addition to pairwise tringularisability such that the origin of (1) is asymptotically stable?

In the sequel we show via a simple example that the condition of pairwise triangularisability is not sufficient to guarantee the existence of a CQLF for each of the constituent sub-systems. In fact, it is readily shown by means of another simple example, and Theorem 2.1, that this condition does not even guarantee asymptotic stability of the origin of Equation (1). However, despite these negative results, we show using state-space-embedding that 
a stable sub-class of pairwise traingularisable systems, of extreme practical importance, can be identified. The usefulness of this sub-class is illustrated in Section 5 .

\section{(i) Common quadratic Lyapunnov functions}

Example 3.1 : Consider the following stable LTI systems,

$$
\Sigma_{A_{i}}: \dot{x}=A_{i} x, A_{i} \in \mathbb{R}^{2 \times 2}
$$

with,

$$
A_{1} \approx\left[\begin{array}{ll}
-1.0000 & 0.0998 \\
-0.9982 & 0.0980
\end{array}\right], A_{2} \approx\left[\begin{array}{cc}
-1.00000 & -0.0995 \\
-0.9945 & -0.1049
\end{array}\right], A_{3} \approx\left[\begin{array}{rr}
-1.0000 & -0.0818 \\
-8.1818 & -1.0000
\end{array}\right] .
$$

The set of matrices for which $A_{i}^{T} P+P A_{i}<0, P=P^{T}>0, P \in \mathbb{R}^{2 \times 2}$, is given by

$$
\mathcal{P}_{A_{i}}: \operatorname{det}\left\{A_{i}^{T} P+P A_{i}\right\}>0
$$

where

$$
P=\left[\begin{array}{cc}
1 & p_{12} \\
p_{12} & p_{22}
\end{array}\right]
$$

Equation (6) defines a convex set in $\left(p_{12}, p_{22}\right)$ space. These sets are depicted in Figure 1 for each of the systems $\Sigma_{A_{1}}, \Sigma_{A_{2}}$, and $\Sigma_{A_{3}}$, as the interior of each of the respective elliptical regions. Clearly, no CQLF exists as the sets $\mathcal{P}_{A_{i}}, i \in[1,2,3]$, have no common point of intersection. However, the matrix pairs $\left\{A_{1}, A_{2}\right\},\left\{A_{2}, A_{3}\right\},\left\{A_{1}, A_{3}\right\}$ are pairwise triangularisable with

$$
T_{12}=\left[\begin{array}{cc}
1 & 0.1 \\
1 & 1
\end{array}\right], T_{23}=\left[\begin{array}{cc}
1 & 1 \\
-10 & 1
\end{array}\right], T_{13}=\left[\begin{array}{cc}
0.1 & 1 \\
1 & 1
\end{array}\right]
$$

(ii) Asymptotic stability of the origin of (1) 


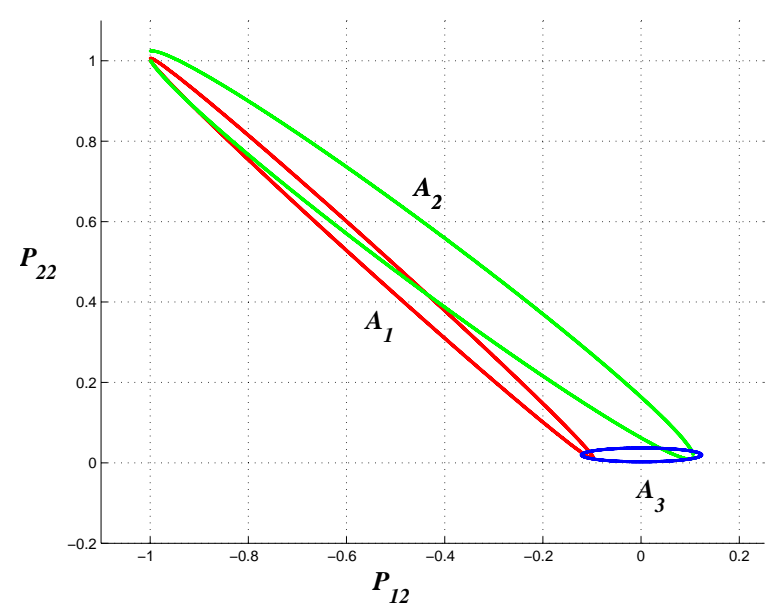

Figure 1: $\mathcal{P}_{A_{i}}$ for $\Sigma_{A_{1}}, \Sigma_{A_{2}}$ and $\Sigma_{A_{3}}$.

It follows from the above example that the condition of pairwise triangulisability of the system matrices does not necessarily imply the existence of a CQLF In fact, the following example demonstrates that the general condition of pairwise triangularisability is not sufficient to guarantee asymptotic stability for the system (1) for arbitrary switching.

Example 3.2 : Consider the following switching system,

$$
\dot{x}=A(t) x, A(t) \in \mathcal{A} \triangleq\left\{A_{1}, A_{2}, A_{3}\right\}
$$

with,

$$
\begin{gathered}
A_{1} \approx\left[\begin{array}{rrr}
-105.2607 & 33.7326 & 65.1648 \\
8.3953 & -6.3633 & -8.3953 \\
-104.7741 & 33.7326 & 64.6782
\end{array}\right], \\
A_{2} \approx\left[\begin{array}{rrr}
-19.4935 & 11.8692 & 18.7430 \\
19.1542 & -12.6197 & -19.1542 \\
-19.0628 & 11.8692 & 18.3124
\end{array}\right], \\
A_{3} \approx\left[\begin{array}{rrr}
0.1333 & -0.0510 & -0.0921 \\
-1.3313 & 0.4788 & 0.8819 \\
1.0659 & -0.3866 & -0.7101
\end{array}\right] .
\end{gathered}
$$


The matrix pairs $\left\{A_{1}, A_{2}\right\},\left\{A_{2}, A_{3}\right\},\left\{A_{1}, A_{3}\right\}$ are pairwise triangularisable with,

$$
\begin{gathered}
T_{12}=\left[\begin{array}{rrr}
2 & 1 & 2 \\
0 & -1 & -11 \\
2 & 1 & 9
\end{array}\right], \\
T_{13}=\left[\begin{array}{rrr}
3 & 2 & 1 \\
-10 & -11 & -10 \\
10 & 9 & 8
\end{array}\right], \\
T_{23}=\left[\begin{array}{rrr}
2 & 1 & 3 \\
-11 & -10 & -10 \\
9 & 8 & 10
\end{array}\right],
\end{gathered}
$$

However, the matrix

$$
\begin{aligned}
A & =0.36 A_{1}+0.24 A_{2}+0.2 A_{3}, \\
& \approx\left[\begin{array}{rrr}
-15.9127 & 4.7986 & 9.5435 \\
-258.6401 & 90.4471 & 168.7670 \\
170.8861 & -62.3351 & -114.3442
\end{array}\right],
\end{aligned}
$$

has an eigenvalue with posive real part $\left(\lambda_{i} \approx-34.9906,0.0169,-4.8360\right)$. It follows from Theorem 2.1, that an unstable switching sequence exists for the system (7). Hence, the general condition of pairwise triangularsability is not a sufficient condition to guarantee asymptotic stability for the system (7). This observation is stated formally as follows.

Proposition 3.1 : Consider the system given by Equation (1). The condition that a set of non-singular matrices $T_{i j}$ exist such that the matrices $\left\{T_{i j} A_{i} T_{i j}^{-1}, T_{i j} A_{j} T_{i j}^{-1}\right\}, i, j, \in$ $\{1, \ldots, m\}$, are upper triangular, is not sufficient to guarantee the asymptotic stability of the system (1).

Proof: The proof is by contradiction and follows directly from Example 3.2. 
(iii) Sufficient conditions for stability

A frequent practical requirement in the design of switching systems is that the nominal closed loop switching-system has real eigenvalues. This requirement ensures that the response of the system does not overshoot. In this context it is of interest to examine pairwise triangularisable systems with the characteristic that all of the matrices in the set $\mathcal{A}$ have real eigenvalues and real eigenvectors. A simple, but non-trivial, pairwise triangularisable system with this property is constructed by choosing $\mathcal{A}$ such that the matrices $A_{i}$ are Hurwitz, and that each pair of matrices share $n-1$ common linearly independent eigenvectors. The matrices specified in Example 3.1 have this property. While these conditions are clearly not sufficient to guarantee the existence of a CQLF, as demonstrated by Example 3.1, it follows via state-space-embedding arguments (Shorten \& Ó Cairbre 2000), and by specifying additional minor technical specifications, that these properties are indeed sufficient to guarantee global attractivity, and hence asymptotic stability (Angeli 1999), of the origin for switching systems of the form of (1). This observation leads to a powerful design technique based upon the following theorem. 
Theorem 3.1.(Shorten \& Ó Cairbre n.d.) Consider the switching system (1) with the set $\mathcal{A}$ defined as follows.

Let $\mathcal{V}=\left\{v_{1}, \ldots, v_{n+1}\right\}$ be a set of real vectors, where each $v_{i} \in \mathbb{R}^{n}$. Suppose any $n$ vectors in $\mathcal{V}$ are linearly independent. For each $i \in\{1,2, \ldots, n+1\}$, construct an $n \times n$ matrix $M_{i}$ as follows: $M_{1}=\left[v_{1}, v_{2}, \ldots, v_{i-1}, v_{i}, \ldots, v_{n-1}, v_{n}\right]$ and for $2 \leq i \leq n+1$ we define $M_{i}=\left[v_{1}, v_{2}, \ldots v_{n+1}, v_{i}, \ldots v_{n-1}, v_{n}\right]$, i.e. $M_{i}$ is obtained by replacing the $(i-1)^{t h}$ column in $M_{1}$ with the vector $v_{n+1}$. Suppose we also have $p$ different diagonal matrices $D_{1}, D_{2}, \ldots, D_{p}$ with all diagonal entries negative. Define $A_{i, h}=M_{i} D_{h} M_{i}^{-1}$, for $1 \leq i \leq n+1$ and $1 \leq h \leq p$. Let $\mathcal{A}$ be a subset of $\left\{A_{i, h}: 1 \leq i \leq n+1,1 \leq h \leq p\right\}$. Then the origin of the system (1) is globally attractive. It further follows from the results in (Angeli 1999), that systems of the form specified above are asymptotically stable.

Comment: The set $\mathcal{A}$ defined in Theorem 3.1 satisfies the following properties:

(a) Every matrix in $\mathcal{A}$ is Hurwitz and diagonalisable;

(b) The eigenvectors of any matrix in $\mathcal{A}$ are real;

(c) Every pair of matrices in $\mathcal{A}$ share at least $n-1$ linearly independent common eigenvectors and can be simultaneously triangularised (Shorten 1996).

Proof of Theorem 3.1: To aid clarity, and to present the arguments in as concise a manner as possible, the reader is now referred to Section 4, where the complete proof of global attractivity of the origin of (1) for a related class of switching system, is presented. The proof of Theorem 3.1 uses state-space-embedding and is presented in full-detail in (Shorten $\&$ Ó Cairbre n.d.) and the Appendix. 


\section{State-space-embedding and a related class of switch- ing system}

An interesting question arises in the context of extending Theorem 3.1 to matrices with complex eigenvectors. These systems are a natural extension of the system class studied in Theorem 3.1. We find that the origin of such systems is globally attractive subject to certain extra minor conditions on the eigenvectors of the matrices. This result is stated formally in Theorem 4.1. The proof of this theorem involves novel extensions to the methods first introduced in (Shorten \& Ó Cairbre 2000), and clearly illustrates the technique of statespace-embedding.

Before proceeding with the main result of this section, we first give an overview of state-spaceembedding. This technique does not involve a quadratic Lyapunov function approach and consequently may be employed in problems where such functions do not exist. State-space embedding essentially comprises the following three steps:

(i) Denote our original switching system by $\dot{x}=A_{i} x$, where $1 \leq i \leq k$. We replace each matrix $A_{i}$ by a matrix $\bar{A}_{i}$ of larger size. The $\bar{A}_{i}^{\prime s}$ are selected so that they all share some common eigenvectors. These $\bar{A}_{i}^{s}$ generate a new switching system which has higher dimension than the original switching system. The new system is denoted by $\dot{\bar{x}}=\bar{A}_{i} \bar{x}$, where $1 \leq i \leq k$.

(ii) We then construct a collection of coordinate systems from various combinations of the eigenvectors of the $\bar{A}_{i}^{s}$. One particular common eigenvector $\tau$ (of all the $\bar{A}_{i}^{\iota^{s}}$ ) will appear as an axis in each one of these coordinate systems. We then consider, in each coordinate system, the projection of the state $\bar{x}(t)$ onto $\tau$ as the dynamics of the new switching system evolves.

(iii) We then consider the convergence of these projections in the long term. For many systems, global attractivity of the origin can be proven by considering convergence properties of these projections. 
We now present the main result of this section; namely Theorem 4.1. The proof of this result is derived using state-space embedding, and clearly illustrates the steps outlined above. 


\section{Preamble for Theorem 4.1:}

Let $\mathcal{V}=\left\{v_{1}, v_{1}^{*}, v_{2}, v_{2}^{*}, \ldots, v_{n+1}, v_{n+1}^{*}\right\}$ be a set of complex (non-real) vectors in $\mathbb{C}^{2 n}$. Here $*$ denotes the complex conjugate. Suppose any $2 n$ vectors of the form $v_{1}, v_{1}^{*}, \ldots, v_{i-1}, v_{i-1}^{*}, v_{i+1}, v_{i+1}^{*}, \ldots, v_{n+1}, v_{n+1}^{*}$, where both $v_{i}$ and $v_{i}^{*}$ are omitted, are linearly independent in $\mathbb{C}^{2 n}$ (over $\mathbb{C}$ ), for any $i \in\{1,2, \ldots, n+1\}$. For each $i \in\{1,2, \ldots, n+1\}$, construct a $2 n \times 2 n$ matrix as follows: $M_{1}=$ $\left[v_{1}, v_{1}^{*}, v_{2}, v_{2}^{*}, \ldots, v_{n}, v_{n}^{*}\right]$ and for $2 \leq i \leq n+1$ we define $M_{i}=\left[v_{1}, v_{1}^{*}, \ldots, v_{n+1}\right.$, $\left.v_{n+1}^{*}, v_{i}, v_{i}^{*}, \ldots, v_{n}, v_{n}^{*}\right]$, i.e. $M_{i}$ is obtained by replacing the $v_{i-1}$ and $v_{i-1}^{*}$ columns by $v_{n+1}$ and $v_{n+1}^{*}$ respectively. Thus, the columns of each $M_{j}$ are linearly independent and so $M_{j}$ is invertible. Suppose we also have $p$ different $2 n \times 2 n$ diagonal matrices $D_{1}, D_{2}, \ldots, D_{p}$ with all diagonal entries negative. In each of these diagonal matrices we assume that the $(k, k)$ entry equals the $(k-1, k-1)$ entry whenever $k$ is even.

Suppose $A_{i, h}=M_{i} D_{h} M_{i}^{-1}$ has real entries for $1 \leq i \leq n+1$ and $1 \leq h \leq p$. In other words, $A_{i, h}$ is a real matrix with complex (non-real) eigenvectors (occurring in conjugate pairs) lying in $\mathcal{V}$ and negative eigenvalues. This gives us $m$ different diagonalisable matrices $A_{i, h}$, where $m=p(n+1)$, because the eigenvectors of $A_{i, h}$ are the columns of $M_{i}$ and the eigenvalues of $A_{i, h}$ are the diagonal entries of $D_{h}$. Let $\mathcal{A}$ be a subset of $\left\{A_{i, h}: 1 \leq i \leq n+1,1 \leq h \leq p\right\}$. Thus, $\mathcal{A}$ is a set of at most $m$ different diagonalisable matrices such that any two matrices in $\mathcal{A}$ have at least $n-1$ linearly independent common conjugate pairs of complex eigenvectors. 
Statement of Theorem 4.1 : If we consider the switching system (1) with the set $\mathcal{A}$ defined as in the above preamble, then the origin is globally attractive. It further follows from the results in (Angeli 1999), that systems of the form specified in Theorem 4.1 are asymptotically stable.

For ease of exposition we first present an outline of the main ideas. This follows closely the three steps of the method of state-space embedding described above. The proof is then developed by means of several key lemmas. Note that in the sequel we use row and column notation interchangeably to denote vectors. This is for convenience of notation.

\section{Outline of Proof:}

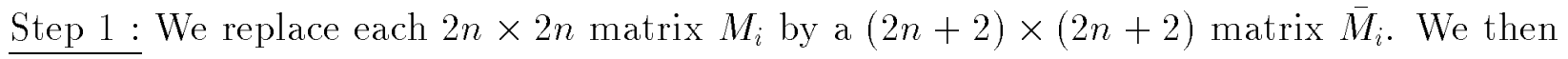
replace each $2 n \times 2 n$ matrix $A_{i, h}$ in $\mathcal{A}$ by a $(2 n+2) \times(2 n+2)$ matrix $\bar{A}_{i, h}$. The matrices $\bar{A}_{i, h} \in \overline{\mathcal{A}}=\left\{\bar{A}_{i, h}: A_{i, h} \in \mathcal{A}\right\}$ are chosen such that there is at least one conjugate pair of common eigenvectors, $\left(a_{1}, a_{2}, 0, \ldots, 0\right),\left(a_{1}^{*}, a_{2}^{*}, 0, \ldots, 0\right)$, for all the matrices in $\overline{\mathcal{A}}$, and also such that the properties of the solutions of the dynamic system,

$$
\dot{\bar{x}}=\bar{A}(t) \bar{x}, \quad \bar{A}(t) \in \overline{\mathcal{A}}
$$

will ultimately imply the global attractivity of the origin of the system,

$$
\dot{x}=A(t) x, \quad A(t) \in \mathcal{A},
$$

where $x=\left(x_{1}, x_{2}, \ldots, x_{2 n}\right)$ and $\bar{x}=\left(x_{2 n+2}, x_{2 n+1}, x_{1}, x_{2}, \ldots, x_{2 n}\right)$.

Step 2 : For a given $j \in\{1,2, \ldots, n+1\}$ we consider the $2 n+2$ linearly independent columns of $\bar{M}_{j}$. These form a $2 n+2$ dimensional coordinate system which includes $\left(a_{1}, a_{2}, 0, \ldots, 0\right)$ as one of the axes. We consider the projection of the state $\bar{x}(t)$ onto $\left(a_{1}, a_{2}, 0, \ldots, 0\right)$ as the dynamics of the system (8) evolve. This projection is given by the first component of the vector,

$$
g_{j}(t)=\bar{M}_{j}^{-1} \bar{x}(t)
$$


and is denoted by $\left[g_{j}\right]_{1}(t)$.

Step 3 : We then show that,

$$
\lim _{t \rightarrow \infty}\left|\operatorname{Re}\left[g_{i}\right]_{1}(t)-\operatorname{Re}\left[g_{j}\right]_{1}(t)\right|=0=\lim _{t \rightarrow \infty}\left|\operatorname{Im}\left[g_{i}\right]_{1}(t)-\operatorname{Im}\left[g_{j}\right]_{1}(t)\right|
$$

for all $i, j \in\{1,2, \ldots, n+1\}$. Note that $R e$ denotes the real part of a complex number and $I m$ denotes the imaginary part. From the above fact we can deduce that $\lim _{t \rightarrow \infty}\left(x_{1}, x_{2}, \ldots, x_{2 n}\right)=$ 0. This is sufficient to demonstrate the global attractivity of the origin of the system,

$$
\dot{x}=A(t) x, A(t) \in \mathcal{A} \text {. }
$$

\section{Technical details of Proof:}

Lemma 4.1 : Suppose $\delta_{2,1}, \delta_{2,2}, \delta_{3,1}, \delta_{3,2}, \ldots, \delta_{n+1,1}, \delta_{n+1,2}$ are any non-zero complex numbers. Then there exist non-zero complex numbers $b_{1}, b_{2}$ such that the set $W=\left\{\left(b_{1}, b_{2} \cdot v_{1}\right)\right.$, $\left.\left(b_{1}^{*}, b_{2}^{*}, v_{1}^{*}\right),\left(\delta_{2,1}, \delta_{2,2}, v_{2}\right),\left(\delta_{2,1}^{*}, \delta_{2,2}^{*}, v_{2}^{*}\right),\left(\delta_{3,1}, \delta_{3,2}, v_{3}\right),\left(\delta_{3,1}^{*}, \delta_{3,2}^{*}, v_{3}^{*}\right), \ldots,\left(\delta_{n+1,1}^{*}, \delta_{n+1,2}^{*}, v_{n+1}^{*}\right)\right\}$ is linearly independent in $\mathbb{C}^{2 n+2}$. Here, $\left(b_{1}, b_{2}, v_{1}\right)$ denotes the vector whose first two coordinates are $b_{1}, b_{2}$ and the remaining $2 n$ coordinates are the $2 n$ coordinates of $v_{1}$.

Proof: We know that $\left\{v_{2}, v_{2}^{*}, \ldots, v_{n+1}, v_{n+1}^{*}\right\}$ is linearly independent in $\mathbb{C}^{2 n}$ and so there exist unique $\alpha_{j}, \beta_{j} \in \mathbb{C}$ such that $v_{1}=\sum_{j=2}^{n+1} \beta_{j} v_{j}+\alpha_{j} v_{j}^{*}$. Pick $b_{2} \in \mathbb{C}$ such that $b_{2} \neq 0$ and $b_{2} \neq$ $\sum_{j=2}^{n+1} \delta_{j, 2} \beta_{j}+\delta_{j, 2}^{*} \alpha_{j}$ We will first prove that the set $Z=\left\{\left(b_{2}, v_{1}\right),\left(\delta_{2,2}, v_{2}\right),\left(\delta_{2,2}^{*}, v_{2}^{*}\right), \ldots,\left(\delta_{n+1,2}^{*}, v_{n+1}^{*}\right)\right\}$ is linearly independent in $\mathbb{C}^{2 n+1}$. Suppose $Z$ is linearly dependent. Thus,

$$
\gamma_{1}\left(b_{2}, v_{1}\right)+\sum_{j=2}^{n+1} \gamma_{j}\left(\delta_{j, 2}, v_{j}\right)+\tau_{j}\left(\delta_{j, 2}^{*}, v_{j}^{*}\right)=0,
$$

for some complex numbers $\gamma_{1}, \gamma_{j}, \tau_{j}, 2 \leq j \leq n+1$ that are not all zero. Hence, $\gamma_{1} \neq 0$ since otherwise $\gamma_{j}=0=\tau_{j}$, for $2 \leq j \leq n+1$. Thus, $v_{1}=-\frac{1}{\gamma_{1}}\left(\sum_{j=2}^{n+1} \gamma_{j} v_{j}+\tau_{j} v_{j}^{*}\right)$, and so $\beta_{j}=-\frac{\gamma_{j}}{\gamma_{1}}$ and $\alpha_{j}=-\frac{\tau_{j}}{\gamma_{1}}$. Also, $\gamma_{1} b_{2}+\sum_{j=2}^{n+1} \gamma_{j} \delta_{j, 2}+\tau_{j} \delta_{j, 2}^{*}=0$. Thus,

$$
b_{2}=-\frac{1}{\gamma_{1}}\left(\sum_{j=2}^{n+1} \gamma_{j} \delta_{j, 2}+\tau_{j} \delta_{j .2}^{*}\right)=\sum_{j=2}^{n+1} \beta_{j} \delta_{j, 2}+\alpha_{j} \delta_{j, 2}^{*},
$$


which is false. Hence, $Z$ is linearly independent in $\mathbb{C}^{2 n+1}$. Now we will show that $W$ is linearly independent in $\mathbb{C}^{2 n+2}$. First note that there exist unique $\epsilon_{1}, \epsilon_{2}, \ldots, \epsilon_{n+1}, \omega_{2}, \omega_{3}, \ldots, \omega_{n+1} \in \mathbb{C}$ such that,

$$
\left(b_{2}^{*}, v_{1}^{*}\right)=\epsilon_{1}\left(b_{2}, v_{1}\right)+\sum_{j=2}^{n+1} \epsilon_{j}\left(\delta_{j, 2}, v_{j}\right)+\omega_{j}\left(\delta_{j, 2}^{*}, v_{j}^{*}\right) .
$$

Pick $b_{1} \in \mathbb{C}$ such that $b_{1} \neq 0$ and $b_{1}^{*}-\epsilon_{1} b_{1} \neq \sum_{j=2}^{n+1} \epsilon_{j} \delta_{j, 1}+\omega_{j} \delta_{j, 1}^{*}$. Suppose $W$ is linearly dependent. Thus,

$$
\zeta_{0}\left(b_{1}^{*}, b_{2}^{*}, v_{1}^{*}\right)+\zeta_{1}\left(b_{1}, b_{2}, v_{1}\right)+\sum_{j=2}^{n+1} \zeta_{j}\left(\delta_{j, 1}, \delta_{j, 2}, v_{j}\right)+\mu_{j}\left(\delta_{j, 1}^{*}, \delta_{j, 2}^{*}, v_{j}^{*}\right)=0,
$$

for some complex numbers $\zeta_{0}, \zeta_{1}, \zeta_{j}, \mu_{j}, 2 \leq j \leq n+1$, that are not all zero. Hence, $\zeta_{0} \neq 0$ since otherwise $\zeta_{1}=\zeta_{j}=\mu_{j}=0,2 \leq j \leq n+1$. Hence,

$$
\left(b_{2}^{*}, v_{1}^{*}\right)=-\frac{1}{\zeta_{0}}\left(\zeta_{1}\left(b_{2}, v_{1}\right)+\sum_{j=2}^{n+1} \zeta_{j}\left(\delta_{j, 2}, v_{j}\right)+\mu_{j}\left(\delta_{j, 2}^{*}, v_{j}^{*}\right)\right),
$$

and so $\epsilon_{1}=-\frac{\zeta_{1}}{\zeta_{0}}, \epsilon_{j}=-\frac{\zeta_{j}}{\zeta_{0}}, \omega_{j}=-\frac{\mu_{j}}{\zeta_{0}}, 2 \leq j \leq n+1$. Also, $\zeta_{0} b_{1}^{*}+\zeta_{1} b_{1}+\sum_{j=2}^{n+1} \zeta_{j} \delta_{j, 1}+\mu_{j} \delta_{j, 1}^{*}=$ 0 , which implies that $b_{1}^{*}-\epsilon_{1} b_{1}=\sum_{j=2}^{n+1} \epsilon_{j} \delta_{j, 1}+\omega_{j} \delta_{j, 1}^{*}$. This is false and so $W$ is linearly independent. Q.E.D.

Define $\bar{M}_{i}$ to be the following $(2 n+2) \times(2 n+2)$ matrix:

$$
\bar{M}_{i}=\left(\begin{array}{cc}
A & H_{i} \\
0 & M_{i}
\end{array}\right) \text {, }
$$

where 0 is the $2 n \times 2$ zero matrix, $M_{i}$ is the $2 n \times 2 n$ matrix defined above, $A=\left(\begin{array}{cc}a_{1} & a_{1}^{*} \\ a_{2} & a_{2}^{*}\end{array}\right)$ is an invertible $2 \times 2$ matrix (and we choose $a_{1}, a_{2}$ to be non-real complex numbers) and finally $H_{i}$ is a $2 \times 2 n$ matrix constructed as follows: If $v_{k}$ forms the $j^{t h}$ column of $M_{i}$, then the $j^{\text {th }}$ column of $H_{i}$ will comprise the first two coordinates of the vector, in $W$ (from Lemma 4.1), containing $v_{k}$. The first coordinate will lie in the first row and the second coordinate will lie in the second row of $H_{i}$. Also, in a similar fashion, the $(j+1)^{s t}$ column of $H_{i}$ will comprise the first two coordinates of the vector, in $W$, containing $v_{k}^{*}$. Thus, for example,

$$
H_{1}=\left(\begin{array}{ccccccc}
b_{1} & b_{1}^{*} & \delta_{2,1} & \delta_{2,1}^{*} & \ldots & \delta_{n, 1} & \delta_{n, 1}^{*} \\
b_{2} & b_{2}^{*} & \delta_{2,2} & \delta_{2,2}^{*} & \ldots & \delta_{n, 2} & \delta_{n, 2}^{*}
\end{array}\right),
$$




$$
\begin{gathered}
H_{2}=\left(\begin{array}{cccccccc}
\delta_{n+1,1} & \delta_{n+1,1}^{*} & \delta_{2,1} & \delta_{2,1}^{*} & \ldots & \delta_{n, 1} & \delta_{n, 1}^{*} \\
\delta_{n+1,2} & \delta_{n+1,2}^{*} & \delta_{2,2} & \delta_{2,2}^{*} & \ldots & \delta_{n, 2} & \delta_{n, 2}^{*}
\end{array}\right), \\
H_{3}=\left(\begin{array}{ccccccccc}
b_{1} & b_{1}^{*} & \delta_{n+1,1} & \delta_{n+1,1}^{*} & \delta_{3,1} & \delta_{3,1}^{*} & \ldots & \delta_{n, 1} & \delta_{n, 1}^{*} \\
b_{2} & b_{2}^{*} & \delta_{n+1,2} & \delta_{n+1,2}^{*} & \delta_{3,2} & \delta_{3,2}^{*} & \ldots & \delta_{n, 2} & \delta_{n, 2}^{*}
\end{array}\right) .
\end{gathered}
$$

Note that,

$$
\bar{M}_{i}^{-1}=\left(\begin{array}{cc}
A^{-1} & -A^{-1} H_{i} M_{i}^{-1} \\
0 & M_{i}^{-1}
\end{array}\right) .
$$

Note that every even row in $\bar{M}_{i}^{-1}$ is the complex conjugate of the previous row. Define $\bar{D}_{h}$ to be the $(2 n+2) \times(2 n+2)$ matrix with zeros everywhere except in the bottom right $2 n \times 2 n$ corner which is occupied by $D_{h}$, i.e.

$$
\bar{D}_{h}=\left(\begin{array}{cc}
0 & 0 \\
0 & D_{h}
\end{array}\right) .
$$

Define $\bar{A}_{i, h}=\bar{M}_{i} \bar{D}_{h} \bar{M}_{i}^{-1}$. We then have that $\bar{A}_{i, h}$ is a $(2 n+2) \times(2 n+2)$ matrix of the form

$$
\bar{A}_{i, h}=\left(\begin{array}{cc}
0 & C_{i, h} \\
0 & A_{i, h}
\end{array}\right),
$$

where $C_{i, h}$ is a $2 \times 2 n$ real matrix, the top left 0 is the $2 \times 2$ zero matrix and the bottom left 0 is the $2 n \times 2$ zero matrix. Note that $\left(a_{1}, a_{2}, 0, \ldots, 0\right),\left(a_{1}^{*}, a_{2}^{*}, 0, \ldots, 0\right)$ form a pair of common eigenvectors for all $m$ matrices $A_{i, h}$. In the following we assume that $x_{1}, x_{2}, \ldots, x_{2 n}, x_{2 n+1}, x_{2 n+2}$ are all real valued functions of $t$.

We then have that,

$$
\left(\begin{array}{c}
\dot{x}_{2 n+2} \\
\dot{x}_{2 n+1} \\
\dot{x}_{1} \\
\dot{x}_{2} \\
\vdots \\
\dot{x}_{2 n}
\end{array}\right)=\bar{A}_{i, h}\left(\begin{array}{c}
x_{2 n+2} \\
x_{2 n+1} \\
x_{1} \\
x_{2} \\
\vdots \\
x_{2 n}
\end{array}\right),
$$


if and only if,

$$
\left(\begin{array}{c}
\dot{x}_{1} \\
\dot{x}_{2} \\
\vdots \\
\dot{x}_{2 n}
\end{array}\right)=A_{i, h}\left(\begin{array}{c}
x_{1} \\
x_{2} \\
\vdots \\
x_{2 n}
\end{array}\right) \text { and } \dot{x}_{2 n+2}=\sum_{j=1}^{2 n} c_{j} x_{j} \text { and } \dot{x}_{2 n+1}=\sum_{j=1}^{2 n} d_{j} x_{j}
$$

for some scalars $c_{j}, d_{j}$. We will now show that $\lim _{t \rightarrow \infty}\left(x_{1}, x_{2}, \ldots, x_{2 n}\right)=0$, for any solution $\left(x_{2 n+2}, x_{2 n+1}, x_{1}, \ldots, x_{2 n}\right)$ to the switching system (8). By the above, this will imply that $\lim _{t \rightarrow \infty}\left(x_{1}, x_{2}, \ldots, x_{2 n}\right)=0$, for any solution $\left(x_{1}, x_{2}, \ldots, x_{2 n}\right)$ to the switching system (1) and that will give us global attractivity of the origin in the switching system (1), and we will be done.

Let $\bar{x}=\left(x_{2 n+2}, x_{2 n+1}, x_{1}, \ldots, x_{2 n}\right)$. We consider the evolution of the system dynamics $(8)$ in each of the coordinate systems,

$$
g_{i}=\bar{M}_{i}^{-1} \bar{x}
$$

There are $n+1$ coordinate systems corresponding to $i \in\{1,2, \ldots, n+1\}$. Each $g_{i}$ is a vector valued function of $t$. Let $\left[g_{i}\right]_{k}$ denote the $k^{t h}$ component of $g_{i}$. Note that if $k$ is even, then $\left[g_{i}\right]_{k}(t)=\left[g_{i}\right]_{k-1}^{*}(t)$. Let $G=\left\{\operatorname{Re}\left[g_{1}\right]_{1}, \operatorname{Re}\left[g_{2}\right]_{1}, \ldots, \operatorname{Re}\left[g_{n+1}\right]_{1}\right\}$. Let the system dynamics be initially described by,

$$
\dot{\bar{x}}=\bar{A}_{j, h} \bar{x}
$$

over some time interval $\left[t_{1}, t_{2}\right]$. Note that $\dot{g}_{j}=\bar{D}_{h} g_{j}$. Denote the $(i, i)$ element in $D_{h}$ by $\psi_{h, i}$. Hence, $\psi_{h, k}=\psi_{h . k-1}$, when $k$ is even. Also, $\psi_{h, i}$ is the $(i+2, i+2)$ element in $\bar{D}_{h}$. Thus, $\left[\dot{g}_{j}\right]_{m}=\psi_{h . m-2}\left[g_{j}\right]_{m}$, for $3 \leq m \leq 2 n+2$ and $\left[\dot{g}_{j}\right]_{1}=0=\left[\dot{g}_{j}\right]_{2}$. Therefore, when we are in system (11), we have,

$$
\left[g_{j}\right]_{m}(t)=\left[g_{j}\right]_{m}\left(t_{1}\right) e^{\psi_{h, m-2}\left(t-t_{1}\right)} \text {, for } 3 \leq m \leq 2 n+2 \text {, }
$$

and $\left[g_{j}\right]_{1},\left[g_{j}\right]_{2}$ are constant functions of $t$. Consider the evolution of $\operatorname{Re}\left[g_{i}\right]_{1}$ relative to $\operatorname{Re}\left[g_{j}\right]_{1}$. This 'distance', denoted by $d_{i, j}(t)$, is given by,

$$
d_{i, j}(t)=\left|\operatorname{Re}\left[g_{i}\right]_{1}(t)-\operatorname{Re}\left[g_{j}\right]_{1}(t)\right|,
$$


and can be conveniently calculated from,

$$
g_{i}=\bar{M}_{i}^{-1} \bar{M}_{j} g_{j}
$$

We now analyse the structure of the matrix $F_{i, j}=\bar{M}_{i}^{-1} \bar{M}_{j}$, for $i \neq j$.

Lemma 4.2: For $i \neq j$, the first two rows of $F_{i, j}$ are of the form,

$$
\left(\begin{array}{ccccccccc}
1 & 0 & 0 & \ldots & \alpha & \beta & 0 & \ldots & 0 \\
0 & 1 & 0 & \ldots & \beta^{*} & \alpha^{*} & 0 & \ldots & 0
\end{array}\right),
$$

where $\alpha, \beta$ depend on $i, j$. At least one of $\alpha, \beta$ is non-zero. $\alpha$ appears in the $k^{\text {th }}$ column, where $k=2 j-1$, if $i=1$, and $k=2 i-1$, if $i>1$. Note also that $k$ is always odd and is never 1.

Proof: Denote the first row of $\vec{M}_{i}^{-1}$ by $\overrightarrow{r_{1}}$ and so the second row is $\vec{r}_{1}^{*}$. Suppose first that $i=1$. We see that a basis for the orthogonal complement of $\overrightarrow{r_{1}}$ in $\mathbb{C}^{2 n+2}$ is given by $\left\{\left(a_{1}, a_{2}, 0\right),\left(b_{1}, b_{2}, v_{1}\right),\left(b_{1}^{*}, b_{2}^{*}, v_{1}^{*}\right),\left(\delta_{2,1}, \delta_{2,2}, v_{2}\right),\left(\delta_{2,1}^{*}, \delta_{2,2}^{*}, v_{2}^{*}\right), \ldots,\left(\delta_{n, 1}^{*}, \delta_{n, 2}^{*}, v_{n}^{*}\right)\right\}$. Hence, by Lemma 4.1, the only places (apart from the first column) in the first row of $F_{i, j}$, which are potentially non-zero, are the $k^{t h}$ and $(k+1)^{s t}$ columns where $k$ is the number of the column in $\bar{M}_{j}$ which has $\left(\delta_{n+1,1}, \delta_{n+1,2}, v_{n+1}\right)$. Thus, $k=2 j-1$. Here, $\alpha$ is the row-column product of $\overrightarrow{r_{1}}$ and $\left(\delta_{n+1,1}, \delta_{n+1,2}, v_{n+1}\right)$. $\beta$ is the row-column product of $\overrightarrow{r_{1}}$ and $\left(\delta_{n+1,1}^{*}, \delta_{n+1,2}^{*}, v_{n+1}^{*}\right)$. Note that at least one of $\alpha, \beta$ is non-zero. Using the fact that the second row of $\bar{M}_{i}^{-1}$ is ${\overrightarrow{r_{1}}}^{*}$, and proceeding as above, we obtain the appropriate second row for $F_{i, j}$.

Suppose next that $i=2$. We see that a basis for the orthogonal complement of $\overrightarrow{r_{1}}$ in $\mathbb{C}^{2 n+2}$ is given by $\left\{\left(a_{1}, a_{2}, 0\right),\left(\delta_{n+1,1}, \delta_{n+1,2}, v_{n+1}\right),\left(\delta_{n+1,1}^{*}, \delta_{n+1,2}^{*}, v_{n+1}^{*}\right),\left(\delta_{2,1}, \delta_{2,2}, v_{2}\right),\left(\delta_{2,1}^{*}, \delta_{2,2}^{*}, v_{2}^{*}\right)\right.$, $\left.\ldots,\left(\delta_{n, 1}^{*}, \delta_{n, 2}^{*}, v_{n}^{*}\right)\right\}$. Hence, by Lemma 4.1, the only places (apart from the first column) in the first row of $F_{i, j}$, which are potentially non-zero, are the $k^{t h}$ and $(k+1)^{\text {st }}$ columns where $k$ is the number of the column in $\bar{M}_{j}$ which has $\left(b_{1}, b_{2}, v_{1}\right)$. Thus, $k=3=2 i-1$. Here, $\alpha$ is the row-column product of $\overrightarrow{r_{1}}$ and $\left(b_{1}, b_{2}, v_{1}\right) . \beta$ is the row-column product of $\overrightarrow{r_{1}}$ and $\left(b_{1}^{*}, b_{2}^{*}, v_{1}^{*}\right)$. Note that at least one of $\alpha, \beta$ is non-zero. Using the fact that the second row of $\bar{M}_{i}^{-1}$ is $\vec{r}_{1}^{*}$, and proceeding as above, we obtain the appropriate second row for $F_{i, j}$. 
Suppose finally that $i \geq 3$. We see that a basis for the orthogonal complement of $\overrightarrow{r_{1}}$ in $\mathbb{C}^{2 n+2}$ is given by deleting $\left(\delta_{i-1,1}, \delta_{i-1,2}, v_{i-1}\right),\left(\delta_{i-1,1}^{*}, \delta_{i-1,2}^{*}, v_{i-1}^{*}\right)$ from $W \cup\left\{\left(a_{1}^{*}, a_{2}^{*}, 0\right)\right\}$ where $W$ is the set defined in Lemma 4.1. Hence, by Lemma 4.1 the only places (apart from the first column) in the first row of $F_{i, j}$, which are potentially non-zero, are the $k^{t h}$ and $(k+1)^{s t}$ columns where $k$ is the number of the column in $\bar{M}_{j}$ which has $\left(\delta_{i-1,1}, \delta_{i-1,2}, v_{i-1}\right)$. Thus, $k=2 i-1$. Here, $\alpha$ is the row-column product of $\overrightarrow{r_{1}}$ and $\left(\delta_{i-1,1}, \delta_{i-1,2}, v_{i-1}\right) . \beta$ is the rowcolumn product of $\overrightarrow{r_{1}}$ and $\left(\delta_{i-1,1}^{*}, \delta_{i-1,2}^{*}, v_{i-1}^{*}\right)$. Note that at least one of $\alpha, \beta$ is non-zero. Using the fact that the second tow of $\bar{M}_{i}^{-1}$ is $\vec{r}_{1}^{*}$, and proceeding as above, we obtain the appropriate second row for $F_{i, j}$. Q.E.D.

Since $\alpha, \beta$, in the above Lemma, depend on $i, j$, we will denote them by $\alpha_{i, j}, \beta_{i, j}$.

We combine Lemma 4.2 with (14) to obtain,

$$
\begin{aligned}
{\left[g_{i}\right]_{1} } & =\left[g_{j}\right]_{1}+\alpha_{i, j}\left[g_{j}\right]_{k}+\beta_{i, j}\left[g_{j}\right]_{k+1} \\
& =\left[g_{j}\right]_{1}+\alpha_{i, j}\left[g_{j}\right]_{k}+\beta_{i, j}\left[g_{j}\right]_{k}^{*}, \text { for } 1 \leq i \leq n+1 \text { with } i \neq j .
\end{aligned}
$$

Note that (15) is true independent of what system we are in. We combine (12) and (15) to obtain,

$$
\operatorname{Re}\left[g_{i}\right]_{1}-\operatorname{Re}\left[g_{j}\right]_{1}=e^{\psi_{h, k-2}\left(t-t_{1}\right)}\left(\operatorname{Re}\left(\alpha_{i, j}\left[g_{j}\right]_{k}\left(t_{1}\right)\right)+\operatorname{Re}\left(\beta_{i, j}\left[g_{j}\right]_{k}^{*}\left(t_{1}\right)\right)\right), \quad \text { for } \quad i \neq j,
$$

whenever we are in system (11). Hence,

$$
d_{i, j}(t)=e^{\psi_{h, k-2}\left(t-t_{1}\right)}\left|\operatorname{Re}\left(\alpha_{i, j}\left[g_{j}\right]_{k}\left(t_{1}\right)\right)+\operatorname{Re}\left(\beta_{i, j}\left[g_{j}\right]_{k}^{*}\left(t_{1}\right)\right)\right|
$$

Consequently, $\frac{d d_{i, j}(t)}{d t}<0$, or else $\operatorname{Re}\left[g_{i}\right]_{1}$ and $\operatorname{Re}\left[g_{j}\right]_{1}$ both agree over the time interval $\left[t_{1}, t_{2}\right]$. Thus, the distance between $\operatorname{Re}\left[g_{i}\right]_{1}(t)$ and the constant $\operatorname{Re}\left[g_{j}\right]_{1}(t)$ is either getting smaller or always zero over the time interval $\left[t_{1}, t_{2}\right]$, when we are in the system described by $\dot{\bar{x}}=\bar{A}_{j, h} \bar{x}$.

Proof of Theorem 4.1 : We will now prove that $\lim _{t \rightarrow \infty}\left(x_{1}, x_{2}, \ldots, x_{2 n}\right)=0$, for any solution $\left(x_{1}, x_{2}, \ldots, x_{2 n}\right)$ to the system (1) with the set $\mathcal{A}$ defined as in the preamble for Theorem 4.1, and then we will be done. Note that,

$$
\left.\mid \operatorname{Re}\left[g_{i}\right]_{1}(t)-\operatorname{Re}\left[g_{j}\right]_{1}\right)(t)\left|=\frac{1}{2}\right| \alpha_{i, j}\left[g_{j}\right]_{k}(t)+\alpha_{i, j}^{*}\left[g_{j}\right]_{k}^{*}(t)+\beta_{i, j}\left[g_{j}\right]_{k}^{*}(t)+\beta_{i, j}^{*}\left[g_{j}\right]_{k}(t) \mid
$$




$$
\begin{aligned}
& =\frac{1}{2}\left|\left(\alpha_{i, j}+\beta_{i, j}^{*}\right)\left[g_{j}\right]_{k}(t)+\left(\alpha_{i, j}^{*}+\beta_{i, j}\right)\left[g_{j}\right]_{k}^{*}(t)\right|, \\
& =\frac{1}{2}\left(r_{i, j} e^{i \theta_{i, j}}\left[g_{j}\right]_{k}\left(t_{1}\right) e^{\psi_{h, k-2}\left(t-t_{1}\right)}+r_{i, j} e^{-i \theta_{i, j}}\left[g_{j}\right]_{k}^{*}\left(t_{1}\right) e^{\psi_{h, k-2}\left(t-t_{1}\right)}\right), \\
& =\left|r_{i, j} \cos \left(\theta_{i, j}+\tau_{j, k, t_{1}}\right)\right|\left|\left[g_{j}\right]_{k}(t)\right|,
\end{aligned}
$$

where $\alpha_{i, j}+\beta_{i, j}^{*}=r_{i, j} e^{i \theta_{i, j}}$ and $\left[g_{j}\right]_{k}\left(t_{1}\right)=s_{j, k, t_{1}} e^{i \tau_{j, k, t_{1}}}$ are polar representations of the appropriate complex numbers. Denote $\left|r_{i, j} \cos \left(\theta_{i, j}+\tau_{j, k, t_{1}}\right)\right|$ by $Q_{i, j, k, t_{1}}$.

Denote the maximum value (minimum value) of $G(t)$, for any time $t$ in the time interval $\left[t_{1}, t_{2}\right]$, by $\max ^{1} G(t)\left(\min ^{1} G(t)\right)$. Recall that we are in system $(11)$ when $t \in\left[t_{1}, t_{2}\right]$. Then,

$$
\begin{aligned}
\max ^{1} G(t)-\min ^{1} G(t) & =\operatorname{Re}\left[g_{i}\right]_{1}(t)-\operatorname{Re}\left[g_{b}\right]_{1}(t), \text { for some } i, b \in\{1,2, \ldots, n+1\} \\
& =\operatorname{Re}\left[g_{i}\right]_{1}(t)-\operatorname{Re}\left[g_{j}\right]_{1}(t)+\operatorname{Re}\left[g_{j}\right]_{1}(t)-\operatorname{Re}\left[g_{b}\right]_{1}(t), \\
& =Q_{i, j, k, t_{1}}\left|\left[g_{j}\right]_{k}\left(t_{1}\right)\right| e^{\psi_{h, k-2}\left(t-t_{1}\right)}+Q_{b, j, q, t_{1}}\left|\left[g_{j}\right]_{q}\left(t_{1}\right)\right| e^{\psi_{h, q-2}\left(t-t_{1}\right)}
\end{aligned}
$$

where, as in Lemma $4.2, k=2 j-1$, if $i=1$, and $k=2 i-1$, if $i>1$. Similarly, $q=2 j-1$, if $b=1$, and $q=2 b-1$, if $b>1$. Note that if $\operatorname{Re}\left[g_{j}\right]_{1}$ is a maximum value (or minimum value) of $G(t)$, then the last line above collapses to just one term instead of two, and in this case the following arguments will also work. Now let $B_{i, j, t_{1}}=Q_{i, j, k, t_{1}}\left|\left[g_{j}\right]_{k}\left(t_{1}\right)\right|=$ distance between $\operatorname{Re}\left[g_{i}\right]_{1}\left(t_{1}\right)$ and $\operatorname{Re}\left[g_{j}\right]_{1}\left(t_{1}\right)$. Let $B_{b, j, t_{1}}=Q_{b, j, q, t_{1}} \mid\left[g_{j}\right]_{q}\left(t_{1}\right)=$ distance between $\operatorname{Re}\left[g_{j}\right]_{1}\left(t_{1}\right)$ and $\operatorname{Re}\left[g_{b}\right]_{1}\left(t_{1}\right)$. Also, let $\lambda=\max \left\{\psi_{h, s}: 1 \leq h \leq p, 1 \leq s \leq 2 n\right\}$. Note that $\lambda<0$. Then,

$$
\begin{aligned}
\max ^{1} G(t)-\min ^{1} G(t) & \leq\left(B_{i, j, t_{1}}+B_{b, j, t_{1}}\right) e^{\lambda\left(t-t_{1}\right)} \\
& \leq\left(\max ^{1} G\left(t_{1}\right)-\min ^{1} G\left(t_{1}\right)\right) e^{\lambda\left(t-t_{1}\right)}
\end{aligned}
$$

The last inequality follows from the fact that, over the time interval $\left[t_{1}, t_{2}\right], \operatorname{Re}\left[g_{i}\right]_{1}(t)$ remains on the same side of the constant $\operatorname{Re}\left[g_{j}\right]_{1}(t)$, and $\operatorname{Re}\left[g_{b}\right]_{1}(t)$ remains on the other side of $\operatorname{Re}\left[g_{j}\right]_{1}(t)$. This is because the right hand side of (16) does not change sign as time changes over the time interval $\left[t_{1}, t_{2}\right]$. Note that $i$ and $b$ may change with time and so $\max ^{1} G\left(t_{1}\right)$ may not correspond to $\operatorname{Re}\left[g_{i}\right]_{1}\left(t_{1}\right)$, and $\min ^{1} G\left(t_{1}\right)$ may not correspond to $\operatorname{Re}\left[g_{b}\right]_{1}\left(t_{1}\right)$. Now suppose we switch to the next (second) system described by,

$$
\dot{\bar{x}}=\bar{A}_{c, w} \bar{x}
$$


over the time interval $\left[t_{2}, t_{3}\right]$. Denote the maximum value (minimum value) of $G(t)$, for any time $t$ in $\left[t_{2}, t_{3}\right]$, by $\max ^{2} G(t)\left(\min ^{2} G(t)\right)$. Then, as above we get,

$$
\begin{aligned}
\max ^{2} G(t)-\min ^{2} G(t) & \leq\left(\max ^{2} G\left(t_{2}\right)-\min ^{2} G\left(t_{2}\right)\right) e^{\lambda\left(t-t_{2}\right)} \\
& \leq\left(\max ^{1} G\left(t_{1}\right)-\min ^{1} G\left(t_{1}\right)\right) e^{\lambda\left(t_{2}-t_{1}\right)} e^{\lambda\left(t-t_{2}\right)}, \\
& =\left(\max ^{1} G\left(t_{1}\right)-\min ^{1} G\left(t_{1}\right)\right) e^{\lambda\left(t-t_{1}\right)}
\end{aligned}
$$

The second inequality above follows from the fact that we start the second system (18) at the time $t_{2}$ when we stop the first system (11), and the initial conditions for the second system are the terminal conditions for the first system at time $t_{2}$. Thus, $\max ^{2} G\left(t_{2}\right)-\min ^{2} G\left(t_{2}\right) \leq$ $\left(\max ^{1} G\left(t_{1}\right)-\min ^{1} G\left(t_{1}\right)\right) e^{\lambda\left(t_{2}-t_{1}\right)}$ from $(17)$.

Now suppose we switch to the next (third) system described by,

$$
\dot{\bar{x}}=\bar{A}_{d, u} \bar{x}
$$

over the time interval $\left[t_{3}, t_{4}\right]$. Denote the maximum value (minimum value) of $G(t)$, for any time $t$ in $\left[t_{3}, t_{4}\right]$, by $\max ^{3} G(t)\left(\min ^{3} G(t)\right)$. Then, as above we get,

$$
\max ^{3} G(t)-\min ^{3} G(t) \leq\left(\max ^{1} G\left(t_{1}\right)-\min ^{1} G\left(t_{1}\right)\right) e^{\lambda\left(t-t_{1}\right)} .
$$

For the general situation, when we have switched for the $m^{\text {th }}$ time, we are in the system described by $\dot{\bar{x}}=\bar{A}_{z, f} \bar{x}$ over the time interval $\left[t_{m}, t_{m+1}\right]$. Again, we denote the maximum value (minimum value) of $G(t)$, for any time $t$ in $\left[t_{m}, t_{m+1}\right]$, by $\max ^{m} G(t)\left(\min ^{m} G(t)\right.$. Then, as above we get,

$$
\max ^{m} G(t)-\min ^{m} G(t) \leq\left(\max ^{1} G\left(t_{1}\right)-\min ^{1} G\left(t_{1}\right)\right) e^{\lambda\left(t-t_{1}\right)} .
$$

Therefore, since $\lambda<0$, we have $\lim _{t \rightarrow \infty}(\max G(t)-\min G(t))=0$, where $\max G(t)(\min G(t))$ denotes the maximum value (minimum value) of $G(t)$ for any time $t \geq t_{1}$. Thus,

$$
\lim _{t \rightarrow \infty}\left|\operatorname{Re}\left[g_{i}\right]_{1}(t)-\operatorname{Re}\left[g_{j}\right]_{1}(t)\right|=0, \text { for all } i, j \in\{1,2, \ldots, n+1\}
$$

Similarly, we can show that,

$$
\lim _{t \rightarrow \infty}\left|\operatorname{Im}\left[g_{i}\right]_{1}(t)-\operatorname{Im}\left[g_{j}\right]_{1}(t)\right|=0, \text { for all } i, j \in\{1,2, \ldots, n+1\} \text {. }
$$


Note that,

$$
\left(\begin{array}{c}
\operatorname{Re}\left[g_{i}\right]_{1}(t)-\operatorname{Re}\left[g_{j}\right]_{1}(t) \\
\operatorname{Im}\left[g_{i}\right]_{1}(t)-\operatorname{Im}\left[g_{j}\right]_{1}(t)
\end{array}\right)=\frac{1}{2}\left(\begin{array}{cc}
\alpha_{i, j}+\beta_{i, j}^{*} & \alpha_{i, j}^{*}+\beta_{i, j} \\
-i\left(\alpha_{i, j}-\beta_{i, j}^{*}\right) & -i\left(\beta_{i, j}-\alpha_{i, j}^{*}\right)
\end{array}\right)\left(\begin{array}{c}
{\left[g_{j}\right]_{k}(t)} \\
{\left[g_{j}\right]_{k}^{*}(t)}
\end{array}\right) .
$$

where the $-i$ is the complex square root of -1 . Denote the above vector on the left hand side by $\Delta_{i, j}(t)$ and denote the above $2 \times 2$ matrix on the right hand side by $X_{i, j}$. Thus,

$$
\Delta_{i, j}(t)=X_{i, j}\left(\begin{array}{c}
{\left[g_{j}\right]_{k}(t)} \\
{\left[g_{j}\right]_{k}^{*}(t)}
\end{array}\right)
$$

We will now show that $X_{i, 1}$ is invertible for $2 \leq i \leq n+1$. First note that

$$
\begin{aligned}
X_{i, j} \text { is invertible } & \Leftrightarrow\left|\beta_{i, j}\right| \neq\left|\alpha_{i, j}\right| \\
& \Leftrightarrow \operatorname{det}\left(\begin{array}{cc}
\alpha_{i, j} & \beta_{i, j} \\
\beta_{i, j}^{*} & \alpha_{i, j}^{*}
\end{array}\right) \neq 0 .
\end{aligned}
$$

Notice how, with a little serving of serendipity, the last matrix above, namely

$$
\left(\begin{array}{cc}
\alpha_{i, j} & \beta_{i, j} \\
\beta_{i, j}^{*} & \alpha_{i, j}^{*}
\end{array}\right)
$$

(which we denote by $W_{i, j}$ ) appears as part of the first two rows of the matrix $F_{i, j}, i \neq j$. We will now exploit this fact. One can show that $F_{i, 1}$ agrees with the identity matrix in every column except the two columns which contain matrix $W_{i, 1}$. Recall that $F_{i, 1}=\bar{M}_{i}^{-1} \bar{M}_{1}$. We will now alter the two matrices $\bar{M}_{i}^{-1}$ and $\bar{M}_{1}$ so that $\operatorname{det} W_{i, 1}$ will appear as a factor in the determinant of an invertible matrix and hence will be non-zero and so $X_{i, 1}$ will be invertible and we will be done. We first swap the last two rows with the first two rows in $\bar{M}_{i}^{-1}$ so that the new first row is the old penultimate row, the new second row is the old last row, the new penultimate row is the old first row and the new last row is the old second row. Denote this new matrix by $S_{i}$. We then replace $\bar{M}_{1}$ by an invertible matrix $T_{i}$ such that certain pairs of columns of $T_{i}$ interact with certain pairs rows of $S_{i}$, to give that $S_{i} T_{i}$ agrees with the identity matrix except in the last two columns and furthermore $W_{i, 1}$ takes up the bottom right hand 
corner of $S_{i} T_{i}$. Consequently,

$$
\operatorname{det} W_{i, 1}=\operatorname{det}\left(S_{i} T_{i}\right) \neq 0
$$

Thus $X_{i, 1}$ is invertible.

Hence, $\lim _{t \rightarrow \infty}\left[g_{1}\right]_{k}(t)=0$, for $3 \leq k \leq 2 n+2$, because $\lim _{t \rightarrow \infty} \Delta_{i, 1}(t)=0$ for $2 \leq i \leq n+1$.

Therefore, since $\lim _{t \rightarrow \infty} \bar{x}(t)=\lim _{t \rightarrow \infty} \bar{M}_{1} g_{1}(t)$, we get,

$$
\begin{aligned}
& \lim _{t \rightarrow \infty}\left(\begin{array}{c}
x_{2 n+2} \\
x_{2 n+1} \\
x_{1} \\
x_{2} \\
\vdots \\
x_{2 n}
\end{array}\right)=\lim _{t \rightarrow \infty}\left(\begin{array}{cc}
A & H_{1} \\
0 & M_{1}
\end{array}\right)\left(\begin{array}{c}
{\left[g_{1}\right]_{1}(t)} \\
{\left[g_{1}\right]_{2}(t)} \\
\vdots \\
{\left[g_{1}\right]_{2 n+2}(t)}
\end{array}\right), \\
& \lim _{t \rightarrow \infty}\left(\begin{array}{c}
x_{1} \\
x_{2} \\
\vdots \\
x_{2 n}
\end{array}\right)=\lim _{t \rightarrow \infty}\left(\begin{array}{ll}
0 & M_{1}
\end{array}\right)\left(\begin{array}{c}
{\left[g_{1}\right]_{1}(t)} \\
{\left[g_{1}\right]_{2}(t)} \\
\vdots \\
{\left[g_{1}\right]_{2 n+2}(t)}
\end{array}\right), \\
& =\left(\begin{array}{c}
0 \\
0 \\
\vdots \\
0
\end{array}\right)
\end{aligned}
$$

Thus,

$$
\lim _{t \rightarrow \infty}\left(x_{1}, x_{2}, \ldots, x_{2 n}\right)=0
$$

and we have global attractivity of the origin in the switching system (1). Q.E.D. 


\section{Example}

As an example of the application of the results presented in the previous sections we consider the design of an automobile speed control system. A simple model for the longtitudinal dynamics of an automobile is given by the following second order linear parameter varying (LPV) structure,

$$
T_{g, v, u}: \frac{d^{2} v}{d t^{2}}+a 1_{g} \frac{d v}{d t}+a 0_{g} v=L_{g} u
$$

where $v \in \mathbb{R}$ is the vehicle velocity, $u \in \mathbb{R}$ the throttle angle, $g \in\{1,2, \cdots, m\}$ is the engaged gear, and where the parameters $a 1_{g}, a 0_{g}, L_{g}$ vary depending on the engaged gear (Shorten 1996). The task of speed regulation requires the design of a control system that not only maintains a constant velocity in the presence of gear changes and road disturbances (rolling hills, inclines, wind resistance, etc.), but that also guarantees stability of the nominal closed loop system. In this section we demonstrate that the results presented in this paper may be used as the basis for a controller design that accommodates all of these design considerations.

The control strategy advocated here consists of a bank of linear controllers (one for each gear) and a switching mechanism that are connected in feedback as depicted in Figure 2. The individual controllers are of the form,

$$
C_{i}: \frac{d u_{i}}{d t}=-b_{i} u_{i}+K 1_{i} e+K 2_{i} \frac{d e}{d t}
$$

where $b_{i} \in \mathbb{R}, K 1_{i} \in \mathbb{R}, K 2_{i} \in \mathbb{R}, r \in \mathbb{R}$ is a constant reference velocity, and where $e=r-v$. A controller of this form is a standard lead-lag controller that is described in elementary text-books (Power \& Simpson 1978). We make the additional assumption that the switching logic selects the appropriate individual controller as the instant of gear change. To keep the discussion as simple as possible we assume that $m=3$, yielding an expression for the closed loop dynamics given by,

$$
\dot{x}=A_{g} x+B r, A_{g} \in\left\{A_{1}, A_{2}, A_{3}\right\},
$$




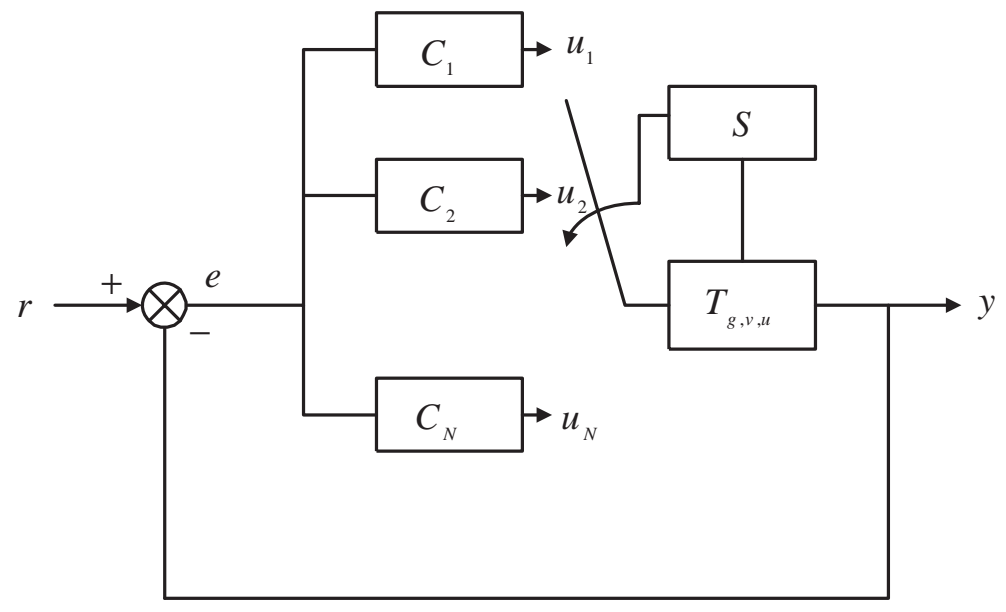

Figure 2: The closed loop switching system. S denotes a switching element

where

$$
\begin{gathered}
x^{T}=\left[v, \frac{d v}{d t}, u_{1}, u_{2}, u_{3}\right], \\
A_{g}=\left[\begin{array}{ccccc}
0 & 1 & 0 & 0 & 0 \\
-a 0_{g} & -a 1_{g} & L_{1} d_{1} & L_{2} d_{2} & L_{3} d_{3} \\
-K 2_{1} & -K 1_{1} & -b_{1} & 0 & 0 \\
-K 2_{2} & -K 1_{2} & 0 & -b_{2} & 0 \\
-K 2_{3} & -K 1_{3} & 0 & 0 & -b_{3}
\end{array}\right], \\
B^{T}=\left[\begin{array}{lllll}
0,0, K 1_{1}, K 1_{2}, & K 1_{3}
\end{array}\right],
\end{gathered}
$$

where $d_{g}=1$ if the $g^{\prime}$ th controller is engaged and zero otherwise. We emphasise that the choice of $m=3$ is motivated by a desire to aid exposition; the arguments and results developed in the sequel generalise to $m$ arbitrary and finite.

Lemma 6.1 : Let $\mathcal{A}=\left\{A_{1}, A_{2}, A_{3}\right\}$ with $A_{g}$ defined by Equation (22). Let the characteristic polynomials of $A_{1}, A_{2}, A_{3}$ be denoted $\Delta_{1}(\lambda), \Delta_{2}(\lambda)$, and $\Delta_{3}(\lambda)$ respectively, with,

$$
\begin{aligned}
& \Delta_{1}(\lambda)=\left(\lambda+b_{3}\right)\left(\lambda+b_{2}\right) H_{1}(\lambda), \\
& \Delta_{2}(\lambda)=\left(\lambda+b_{3}\right)\left(\lambda+b_{1}\right) H_{2}(\lambda), \\
& \Delta_{3}(\lambda)=\left(\lambda+b_{1}\right)\left(\lambda+b_{2}\right) H_{3}(\lambda),
\end{aligned}
$$


where

$$
H_{g}(\lambda)=\lambda^{3}+\left(a 1_{g}+b_{g}\right) \lambda^{2}+\left(a 0_{g}+a 1_{g} b_{g}+L_{g} K 1_{g}\right) \lambda+L_{g} K 2_{g}+a 0_{g} b_{g} .
$$

We choose the $K_{1} g, K 2_{g}, b_{g}$ such that $H_{1}(\lambda)=H_{2}(\lambda)=H_{3}(\lambda)=H(\lambda)$ for all $\lambda$. We consider the case where the roots of the polynomial $P(\lambda)=\left(\lambda+b_{3}\right)\left(\lambda+b_{2}\right)\left(\lambda+b_{1}\right) H(\lambda)$ are distinct. Then the matrices $A_{1}, A_{2}$ and $A_{3}$ have exactly $n-1$ linearly independent common eigenvectors.

Proof : We show the result for $A_{1}$ and $A_{2}$ (identical arguments can be developed for the matrix pairs $\left(A_{1}, A_{3}\right)$ and $\left.\left(A_{2}, A_{3}\right)\right)$ :

$$
\begin{gathered}
A_{1}=\left[\begin{array}{ccccc}
0 & 1 & 0 & 0 & 0 \\
-a 0_{1} & -a 1_{1} & L_{1} & 0 & 0 \\
-K 2_{1} & -K 1_{1} & -b_{1} & 0 & 0 \\
-K 2_{2} & -K 1_{2} & 0 & -b_{2} & 0 \\
-K 2_{3} & -K 1_{3} & 0 & 0 & -b_{3}
\end{array}\right], \\
A_{2}=\left[\begin{array}{ccccc}
0 & 1 & 0 & 0 & 0 \\
-K 0_{2} & -a 1_{2} & 0 & L_{2} & 0 \\
-K 2_{1} & -K 1_{1} & -b_{1} & 0 & 0 \\
-K 2_{3} & -K 1_{2} & 0 & -b_{2} & 0 \\
-K 1_{3} & 0 & 0 & -b_{3}
\end{array}\right] .
\end{gathered}
$$

We need to show that for each common eigenvalue, the matrices $A_{1}$ and $A_{2}$ have a common eigenvector, and that for the eigenvalue that is not common, the matrices have no common eigenvector. We also note that, by definition, $A_{1}$ and $A_{2}$ have $n-1$ distinct common eigenvalues. These eigenvalues correspond to the roots of $H(\lambda)$ and $\lambda=-b_{3}$. The eigenvalues $\lambda=-b_{1}$ (corresponding to $A_{1}$ ) and $\lambda=-b_{2}$ (corresponding to $A_{2}$ ) are not common to both matrices. 
Common eigenvalues:

It follows from the form of $A_{1}$ and $A_{2}$ that the common eigenvector that corresponds to $\lambda=-b_{3}$ is given by $v_{1}^{T}=[0,0,0,0,1]$.

Let $\lambda$ be an eigenvalue that is common to both matrices that is not equal to $-b_{3}$. The eigenvector of $A_{1}$ that corresponds to the eigenvalue $\lambda$ can be obtained by determining the null space of $\lambda I-A_{1}$ :

$$
\lambda I-A_{1}=\left[\begin{array}{ccccc}
\lambda & -1 & 0 & 0 & 0 \\
a 0_{2} & \lambda+a 1_{2} & L_{1} & 0 & 0 \\
K 2_{1} & K 1_{1} & \lambda+b_{1} & 0 & 0 \\
K 2_{2} & K 1_{2} & 0 & \lambda+b_{2} & 0 \\
K 2_{3} & K 1_{3} & 0 & 0 & \lambda+b_{3}
\end{array}\right] .
$$

Let $\left[r A 1_{1}, r A 1_{2}, r A 1_{3}, r A 1_{4}, r A 1_{5}\right]$ denote the row vectors of the matrix $\lambda I-A_{1}$, where $r A 1_{i}$ is the $i$ 'th row vector. From the above discussion $\lambda \neq b \in\left\{-b_{1},-b_{2},-b_{3}\right\}$. Hence, it immediately follows that the row vectors $\left[r A 1_{1}, r A 1_{3}, r A 1_{4}, r A 1_{5}\right]$ are linearly independent. However, the matrix $\lambda I-A_{1}$ is singular. Hence, it must be possible to write $r A 1_{2}$ as a linear combination of $\left[r A 1_{1}, r A 1_{3}, r A 1_{4}, r A 1_{5}\right]$. This implies that the eigenvector corresponding to $\lambda$, is completely specified by the vectors $\left[r A 1_{1}, r A 1_{3}, r A 1_{4}, r A 1_{5}\right]$.

Now consider the matrix $A_{2}$. The eigenvector of $A_{1}$ that corresponds to the eigenvalue $\lambda$ can be obtained by determining the null space of $\lambda I-A_{2}$ :

$$
\lambda I-A_{2}=\left[\begin{array}{ccccc}
\lambda & -1 & 0 & 0 & 0 \\
a 0_{2} & \lambda+a 1_{2} & 0 & L_{2} & 0 \\
K 2_{1} & K 1_{1} & \lambda+b_{1} & 0 & 0 \\
K 2_{2} & K 1_{2} & 0 & \lambda+b_{2} & 0 \\
K 2_{3} & K 1_{3} & 0 & 0 & \lambda+b_{3}
\end{array}\right] .
$$

As before, let $\left[r A 2_{1}, r A 2_{2}, r A 2_{3}, r A 2_{4}, r A 2_{5}\right]$ denote the row vectors of the matrix $\lambda I-$ 
$A_{2}$, where $r A 2_{i}$ is the $i$ 'th row vector. Again, $\lambda \neq b \in\left\{-b_{1},-b_{2},-b_{3}\right\}$. Hence, the row vectors $\left[r A 2_{1}, r A 2_{3}, r A 2_{4}, r A 2_{5}\right]$ are linearly independent. However, as the matrix $\lambda I-A_{2}$ is singular, it must again be possible to write $r A 2_{2}$ as a linear combination of $\left[r A 2_{1}, r A 2_{3}, r A 2_{4}, r A 2_{5}\right]$. This implies that the eigenvector corresponding to $\lambda$, is completely specified by the vectors $\left[r A 2_{1}, r A 2_{3}, r A 2_{4}, r A 2_{5}\right]$.

But, the matrices $A_{1}$ and $A_{2}$ are identical except for the second row. Hence, it follows that $r A 1_{i}=r A 2_{i}, \forall i=\{1,3,4,5\}$, and that the matrices $A_{1}$ and $A_{2}$ have a common eigenvector for all common eigenvalues $\lambda, \lambda \neq b \in\left\{-b_{1},-b_{2},-b_{3}\right\}$. Q.E.D.

Eigenvalues that are not common to both matrices:

Consider the matrix $A_{1}$. The eigenvalue of $A_{1}$ that is not common to $A_{2}$ is $-b_{2}$. The eigenvector of $A_{1}$ that corresponds to this eigenvalue is $v_{1}^{T}=[0,0,0,1,0]$. Now consider the matrix $A_{2}$. The eigenvalue of $A_{2}$ that is not common to $A_{1}$ is $-b_{1}$. The eigenvector of $A_{2}$ that corresponds to this eigenvalue is $v_{2}^{T}=[0,0,1,0,0]$. Clearly, $v_{1} \neq v_{2}$. Q.E.D.

We now note the following facts concerning the matrix $A_{g}$.

(i) $\operatorname{rank}\left\{A_{i}-A_{j}\right\}=1, i \neq j, i, j \in\{1,2,3\}$.

(ii) The characteristic polynomials $\Delta_{1}(\lambda), \Delta_{2}(\lambda)$, and $\Delta_{3}(\lambda)$ share $n-1$ common eigenvalues if $H_{i}(\lambda)=H(\lambda), i \in\{1,2,3\}$.

(iii) Let $H_{i}(\lambda)=H(\lambda), i \in\{1,2,3\}$. Then the matrices $A_{i}$ and $A_{j}, i \neq j, i, j \in\{1,2,3\}$ satisfy Lemma 6.1 and share $n-1$ common real linearly independent eigenvectors.

Therefore, sufficient conditions for the matrices $A_{g} \in\left\{A_{1}, A_{2}, A_{3}\right\}$ to satisfy the conditions of Theorem 3.1, and hence for the stability of the unforced system,

$$
\dot{x}=A_{g} x, g \in\left\{A_{1}, A_{2}, A_{3}\right\}
$$

are given by: 
(i) the target polynomials $H_{i}(\lambda)$ have real negative eigenvalues for all $i \in\{1,2,3\}$;

(ii) $H_{i}(\lambda)=H_{j}(\lambda), i, j \in\{1,2,3\}$;

(iii) $b_{i}>0, i \in\{1,2,3\}$;

(iv) the roots of the polynomial $P(\lambda)=\left(\lambda+b_{3}\right)\left(\lambda+b_{2}\right)\left(\lambda+b_{1}\right) H(\lambda)$ are distinct.

When these conditions are satisfied, one can easily verify that any 5 of the 6 linearly independent eigenvectors given by the eigenvectors of $A_{1}, A_{2}, A_{3}$, are linearly independent. Therefore, the hypothesis of Theorem 3.1 is satisfied, and the origin of (29) is globally attractive and asymptotically stable. BIBO (bounded-input bounded-output) stability of the forced system (22) follows directly from elementary arguments (see (Rugh 1996)).

\section{Concluding remarks}

In this paper we have shown global attractivity for several classes of switching systems. These results were derived using a technique know as state-space embedding. This technique does not rely on the existence of a CQLF, and can therefore be used in situations where such a function does not exist. It is likely that derived methodology is applicable to a wide class of related switching systems. We have also shown that the condition of pairwise triangularisability is not a sufficient condition for the existence of a CQLF, or for the stability of the switching system. However, the derived results also suggest that by imposing additional minor assumptions, the condition of simultaneous triangularisability may be relaxed significantly without the loss of asymptotic stability.

\section{Acknowledgements}

This work was partially supported by the European Union funded research training network Multi-Agent Control, HPRN-CT-1999-00107 and by the Enterprise Ireland grant SC/2000/084/Y. 
This work is the sole responsibility of the authors and does not reflect the European Union's opinion. The $\mathrm{EU}$ is not responsible for any use of data appearing in this publication. The second author gratefully acknowledges many discussions with Dr. Douglas Leith and Dr. Paul Curran. 


\section{Appendix}

\section{Proof of Theorem 2.1}

In order to demonstrate the existence of an unstable switching sequence for Equation (1), it is sufficient to show the existence of a periodic switching sequence for which the state of the system becomes unbounded.

Consider the system (1) with $A(t)=A(t+T)$ for all $t>0$, where $\mathrm{T}$ is the fixed period. Further, let the matrices $\left\{A_{1}, A_{2}, \ldots, A_{M}\right\}$ be indexed according to the order in which they describe the system dynamics, and let the M-tuple $\left\{t_{1}, t_{2}, \ldots, t_{M}\right\}$ describe the time for which each of the matrices describes the system dynamics, i.e., $\dot{x}=A_{j} x$, describes the evolution of the system dynamics for $\left(\sum_{i=1}^{j-1} t_{i}\right) \leq t \leq\left(\sum_{i=1}^{j} t_{i}\right)$. The solution to Equation (1) at time $T=\sum_{i=1}^{M} t_{i}$, is given by,

$$
\begin{aligned}
x(T) & =\left(e^{A_{i} t_{1}} e^{A_{j} t_{2}} \ldots e^{A_{M} t_{M}}\right) x_{0}, \\
& =\Phi(T) x_{0},
\end{aligned}
$$

where $x_{0} \in \mathbb{R}^{N}$ is some initial condition. A sufficient condition for instability of (1) is that the matrix $\Phi(T)$ has an eigenvalue whose magnitude is greater than unity (Mohler 1991). Equation (25) can be expanded as a power series,

$$
\begin{aligned}
\Phi(T) & =I+\left(\sum_{i=1}^{M} \alpha_{i} A_{i}\right) T+K_{2} T^{2}+\ldots \\
& =I+\sigma\left[A_{i}, M\right] T+\ldots
\end{aligned}
$$

where $\alpha_{i}=\frac{t_{i}}{T}, K_{2} \in \mathbb{R}^{N \times N}$, and where we define $\sigma\left[A_{i}, M\right] .=\sum_{i=1}^{M} \alpha_{i} A_{i}$. The expansion (26) is the product of $M$ absolutely convergent series, and is analytic in $T$. Hence, from Theorem 2.1 in (Shorten et al. 2000) and (Kato 1980) (page 81), it follows that, given any eigenvalue $a_{j}$ of $\sigma\left[A_{i}, M\right]$, an eigenvalue of $\Phi(T)$ can be written,

$$
\lambda_{j}(T)=1+a_{j} T+f(T)
$$

where $f(T)=O\left(T^{\beta}\right), \beta>1$, as $T \rightarrow 0$. Thus, there exist constants $C, \epsilon$ such that

$$
|f(T)| \leq C T^{\beta} \text {, for } 0<T<\epsilon .
$$


Hence, $|\operatorname{Re}(f(T))| \leq C T^{\beta}$ for $0<T<\epsilon$, where $\operatorname{Re}(z)$ denotes the real part of a complex number $z$. Now consider the case when the $j^{\text {th }}$ eigenvalue, $a_{j}$, of the matrix $\sigma\left[A_{i}, M\right]$, has a positive real part for some $\left\{\alpha_{1}, \ldots, \alpha_{M}\right\}, \alpha_{i} \geq 0, \sum_{i=1}^{M} \alpha_{i}>0$. Hence, there exists $\delta<\epsilon$, such that for $0<T<\delta$, we have the following:

$$
\begin{aligned}
C T^{\beta} & <\operatorname{Re}\left(a_{j}\right) T, \\
|\operatorname{Re}(f(T))| & <\operatorname{Re}\left(a_{j}\right) T, \\
\operatorname{Re}\left(a_{j}\right) T+\operatorname{Re}(f(T)) & >0, \\
\operatorname{Re}\left(1+a_{j} T+f(T)\right) & >1, \\
\operatorname{Re}\left(\lambda_{j}(T)\right) & >1 . \text { Q.E.D. }
\end{aligned}
$$




\section{Proof of Theorem 3.1.}

For ease of exposition we first present an outline of the main ideas. This follows closely the three steps of the method of state-space embedding described in the main text. The proof is then developed by means of several key lemmas. Note that in the sequel we use row and column notation interchangeably to denote vectors.

\section{Outline of Proof}

Step 1: We replace each $n \times n$ matrix $M_{j}$ by an $(n+1) \times(n+1)$ matrix $\bar{M}_{j}$. We then replace each $n \times n$ matrix $A_{i, h}$ in $\mathcal{A}$ by an $(n+1) \times(n+1)$ matrix $\bar{A}_{i, h}$. The matrices $\bar{A}_{i, h} \in \overline{\mathcal{A}}=\left\{\bar{A}_{i, h}: A_{i, h} \in \mathcal{A}\right\}$ are chosen such that there is at least one common eigenvector $\tau=(1,0,0, \ldots, 0)$ for all the matrices in $\overline{\mathcal{A}}$, and also such that the properties of the solutions of the dynamic system

$$
\dot{\bar{x}}=\bar{A}(t) \bar{x}, \bar{A}(t) \in \overline{\mathcal{A}}
$$

will ultimately imply the global attractivity of the origin of the system

$$
\dot{x}=A(t) x, A(t) \in \mathcal{A}
$$

where $x=\left(x_{1}, \ldots, x_{n}\right)$ and $\bar{x}=\left(x_{n+1}, x_{1}, \ldots, x_{n}\right)$. 
Step 2: For a given $j \in\{1,2, \ldots, n+1\}$ we consider the $n+1$ linearly independent columns of $\bar{M}_{j}$. These form an $n+1$ dimensional coordinate system which includes $\tau$ as one of the axes. We consider the projection of the state $\bar{x}(t)$ onto $\tau$ as the dynamics of the system (29) evolve. This projection is given by the first component of the vector

$$
g_{j}(t)=\bar{M}_{j}^{-1} \bar{x}(t)
$$

and is denoted by $\left[g_{j}\right]_{1}(t)$.

Step 3: We then show that $\lim _{t \rightarrow \infty}\left|\left[g_{j}\right]_{1}(t)-\left[g_{i}\right]_{1}(t)\right|=0, \forall i, j \in\{1, \ldots, n+1\}$. From this fact we can deduce that $\lim _{t \rightarrow \infty}\left(x_{1}, \ldots, x_{n}\right)=0$. This is sufficient to demonstrate the global attractivity of the origin of the system,

$$
\dot{x}=A(t) x, A(t) \in \mathcal{A}
$$

\section{Technical details of Proof}

\section{Lemma 3.1.}

There exists a positive number $a$ such that the set $W=\left\{\left(a, v_{1}\right),\left(1, v_{2}\right),\left(1, v_{3}\right), \ldots,\left(1, v_{n+1}\right)\right\}$ is linearly independent in $\mathbb{R}^{n+1}$. Here $\left(a, v_{1}\right)$ is the vector with $n+1$ coordinates, whose first coordinate is $a$ and remaining $n$ coordinates are the $n$ coordinates of $v_{1}$.

\section{Proof :}

Let $\mathcal{V}=\left\{v_{1}, v_{2}, \ldots, v_{n+1}\right\}$ in $\mathbb{R}^{n}$. We know that any subset of $\mathcal{V}$ which contains $n$ elements is linearly independent and thus forms a basis for $\mathbb{R}^{n}$. Consequently, $\left\{v_{2}, v_{3}, \ldots, v_{n+1}\right\}$ forms a basis for $\mathbb{R}^{n}$ and so there exist unique real numbers $\beta_{j}$ such that $v_{1}=\sum_{j=2}^{n+1} \beta_{j} v_{j}$. Pick $a$ to be a positive number which is different from $\sum_{j=2}^{n+1} \beta_{j}$.

We now show that the set $W$ is linearly independent in $\mathbb{R}^{n+1}$. Let $\bar{v}_{1}=\left(a, v_{1}\right)$ and $\bar{v}_{j}=$ $\left(1, v_{j}\right)$, for $2 \leq j \leq n+1$. Suppose $\sum_{i=1}^{n+1} \gamma_{i} \bar{v}_{i}=0$ with at least one of the $\gamma_{i}^{\prime s}$ non-zero. We 
want to derive a contradiction. Note that $\gamma_{1} a+\sum_{j=2}^{n+1} \gamma_{j}=0$ and $\sum_{i=1}^{n+1} \gamma_{i} v_{i}=0$. Also note that $\gamma_{1} \neq 0$, because if $\gamma_{1}=0$ then we have $\sum_{j=2}^{n+1} \gamma_{j} v_{j}=0$ and so $\gamma_{i}=0$ for $1 \leq i \leq n+1$ and this is false. Consequently, we can write $v_{1}=-\frac{1}{\gamma_{1}} \sum_{j=2}^{n+1} \gamma_{j} v_{j}$ and $a=-\frac{1}{\gamma_{1}} \sum_{j=2}^{n+1} \gamma_{j}$. Thus by uniqueness of $\beta_{j}$, we have $\beta_{j}=-\frac{\gamma_{j}}{\gamma_{1}}$, for $2 \leq j \leq n+1$ and so $a=\sum_{j=2}^{n+1} \beta_{j}$, which is false. Therefore $W$ is linearly independent in $\mathbb{R}^{n+1}$. Q.E.D.

Define $\bar{M}_{i}$ to be the following $(n+1) \times(n+1)$ matrix:

$$
\bar{M}_{i}=\left(\begin{array}{cccccc}
1 & b & 1 & 1 & \ldots & \ldots 1 \\
0 & & & & \\
0 & & M_{i} & & \\
0 & & & & \\
\vdots & & & & \\
0 & & & &
\end{array}\right)
$$

where $b=a$ (from Lemma 3.1), if $i \neq 2$ and $b=1$, if $i=2$. The change in the value of $b$ is because $v_{1}$ only appears in $M_{i}$ when $i \neq 2$. Note that the columns of $\bar{M}_{i}$, apart from the first column, are vectors from the set $W$ in Lemma 3.1 .

Note that

$$
\bar{M}_{i}^{-1}=\left(\begin{array}{ccccc}
1 & s_{1} & s_{2} & \ldots & s_{n} \\
0 & & & & \\
0 & M_{i}^{-1} & & \\
\vdots & & & \\
0 & & &
\end{array}\right)
$$

for some real numbers $s_{1}, s_{2}, \ldots, s_{n}$ which depend on $i$. 
Define $\bar{D}_{h}$ to be the following $(n+1) \times(n+1)$ diagonal matrix:

$$
\bar{D}_{h}=\left(\begin{array}{ccccc}
0 & \ldots & \ldots & \ldots & 0 \\
0 & & & & \\
0 & & D_{h} & & \\
0 & & & & \\
\vdots & & & \\
0 & & &
\end{array}\right)
$$

Define $\bar{A}_{i, h}=\bar{M}_{i} \bar{D}_{h} \bar{M}_{i}^{-1}$. We then get

$$
\bar{A}_{i, h}=\left(\begin{array}{ccccc}
0 & c_{1} & c_{2} & \ldots & c_{n} \\
0 & & & & \\
0 & & A_{i, h} & & \\
\vdots & & & & \\
0 & & &
\end{array}\right)
$$

for some real numbers $c_{1}, c_{2}, \ldots, c_{n}$ which depend on $i$ and $h$. Note that $(1,0,0, \ldots, 0)$ is a common eigenvector for all the $m$ matrices $\bar{A}_{i, h}$.

We then have that

$$
\left(\begin{array}{c}
\dot{x}_{n+1} \\
\dot{x}_{1} \\
\dot{x}_{2} \\
\vdots \\
\dot{x}_{n}
\end{array}\right)=\bar{A}_{i, h}\left(\begin{array}{c}
x_{n+1} \\
x_{1} \\
x_{2} \\
\vdots \\
x_{n}
\end{array}\right)
$$

if and only if

$$
\left(\begin{array}{c}
\dot{x}_{1} \\
\dot{x}_{2} \\
\vdots \\
\dot{x}_{n}
\end{array}\right)=A_{i, h}\left(\begin{array}{c}
x_{1} \\
x_{2} \\
\vdots \\
x_{n}
\end{array}\right) \text { and } \dot{x}_{n+1}=\sum_{i=1}^{n} c_{i} x_{i}
$$

We will show that $\lim _{t \rightarrow \infty}\left(x_{1}, x_{2}, \ldots, x_{n}\right)=0$, for any solution $\left(x_{n+1}, x_{1}, x_{2}, \ldots, x_{n}\right)$ to the switching system (29). By the above, this will then imply that $\lim _{t \rightarrow \infty}\left(x_{1}, x_{2}, \ldots, x_{n}\right)=0$, 
for any solution $\left(x_{1}, x_{2}, \ldots, x_{n}\right)$ to the switching system (1) and that will give us global attractivity of the origin in the switching system (1), and we will be done.

Let $\bar{x}=\left(x_{n+1}, x_{1}, x_{2}, \ldots, x_{n}\right)$. We consider the evolution of the system dynamics $(29)$ in each of the coordinate systems

$$
g_{i}=\bar{M}_{i}^{-1} \bar{x}
$$

There are $n+1$ coordinate systems corresponding to $i \in\{1,2, \ldots, n+1\}$. Let $G=$ $\left\{\left[g_{1}\right]_{1},\left[g_{2}\right]_{1}, \ldots,\left[g_{n+1}\right]_{1}\right\}$, where $\left[g_{i}\right]_{1}$ denotes the first component of the vector $g_{i}$.

Let the system dynamics be initially described by

$$
\dot{\bar{x}}=\bar{A}_{j, h} \bar{x}
$$

over some time interval $\left[t_{1}, t_{2}\right]$. Note that $\dot{g}_{j}=\bar{D}_{h} g_{j}$. Let $\left[g_{j}\right]_{m}$ be the $m^{\text {th }}$ component of the vector $g_{j}$ and let $\lambda_{h, i}$ denote the $(i, i)^{t h}$ diagonal entry in $D_{h}$ and hence the $(i+1, i+1)^{t h}$ diagonal entry in $\bar{D}_{h}$. Then we have that $\left[\dot{g}_{j}\right]_{m}=\lambda_{h, m-1}\left[g_{j}\right]_{m}$, for $m \neq 1$ and $\left[\dot{g}_{j}\right]_{1}=0$. Therefore, when we are in system (35), we have

$$
\left[g_{j}\right]_{m}(t)=\left[g_{j}\right]_{m}\left(t_{1}\right) e^{\lambda_{h, m-1}\left(t-t_{1}\right)}, \text { for } m \neq 1
$$

and $\left[g_{j}\right]_{1}$ is a constant function of $t$.

The members of $G$, when we are in system (35), are illustrated in Figure 1 . Note that $\left[g_{j}\right]_{1}$ is a constant function of time over $\left[t_{1}, t_{2}\right]$ while the other $\left[g_{j}\right]_{m}$ 's vary with time according to $(36)$.

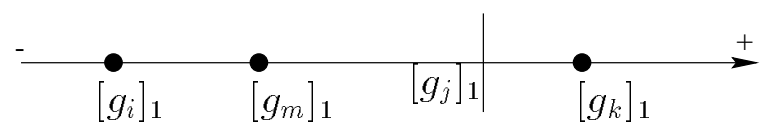

Figure 3: Members of the set $G$.

Consider the evolution of $\left[g_{i}\right]_{1}$ relative to $\left[g_{j}\right]_{1}$. This 'distance' denoted by $d_{i, j}(t)$, is given by

$$
d_{i, j}(t)=\left|\left[g_{i}\right]_{1}(t)-\left[g_{j}\right]_{1}(t)\right|
$$


and can be conveniently calculated from

$$
g_{i}=\bar{M}_{i}^{-1} \bar{M}_{j} g_{j}
$$

We now analyse the structure of the matrix $F_{i, j}=\bar{M}_{i}^{-1} \bar{M}_{j}$, for $i \neq j$. We see that 1 always appears in the first row first column entry of $F_{i, j}$. We claim that there is only one other non-zero entry in the first row.

\section{Lemma 3.2.}

If we exclude the first column of the matrix $F_{i, j}$, for $i \neq j$, then there is only one non-zero entry (denoted by $C_{i, j, k}$ ) in the first row. $C_{i, j, k}$ appears in the $k^{\text {th }}$ column where $k=j$, when $i=1$, and $k=i$, when $i \neq 1$. Note that $k$ is never 1 .

\section{Proof :}

Denote the first row of $\bar{M}_{i}^{-1}$ by $\vec{r}$. Suppose first that $i=1$. We see that a basis for the orthogonal complement of $\vec{r}$ in $\mathbb{R}^{n+1}$ is given by $\left\{\left(a, v_{1}\right),\left(1, v_{2}\right),\left(1, v_{3}\right), \ldots,\left(1, v_{n}\right)\right\}$. Hence, using the result of Lemma 3.1, the only place (apart from the first column) in the first row of $F_{i, j}$ which is non-zero, is the $k^{t h}$ column where $k$ is the number of the column in $\bar{M}_{j}$ which has $\left(1, v_{n+1}\right)$. Thus $k=j$. Here $C_{i, j, k}$ is the dot product of $\vec{r}$ and $\left(1, v_{n+1}\right)$.

Suppose next that $i=2$. We see that a basis for the orthogonal complement of $\vec{r}$ in $\mathbb{R}^{n+1}$ is given by $\left\{\left(1, v_{2}\right),\left(1, v_{3}\right), \ldots,\left(1, v_{n+1}\right)\right\}$. Hence, as above, the only place (apart from the first column) in the first row of $F_{i, j}$ which is non-zero, is the $k^{\text {th }}$ column where $k$ is the number of the column in $\bar{M}_{j}$ which has $\left(a, v_{1}\right)$. Thus $k=2$. Here $C_{i, j, k}$ is the dot product of $\vec{r}$ and $\left(a, v_{1}\right)$.

Suppose finally that $i>2$. We see that a basis for the orthogonal complement of $\vec{r}$ in $\mathbb{R}^{n+1}$ is obtained by deleting $\left(1, v_{i-1}\right)$ from the set $W$ in Lemma 3.1 . Hence, as above, the only place (apart from the first column) in the first row of $F_{i, j}$ which is non-zero, is the $k^{\text {th }}$ column where $k$ is the number of the column in $\bar{M}_{j}$ which has $\left(1, v_{i-1}\right)$. Thus $k=i$. Here $C_{i, j, k}$ is the dot product of $\vec{r}$ and $\left(1, v_{i-1}\right)$. Q.E.D. 
We combine Lemma 3.2 with (38) to obtain

$$
\left[g_{i}\right]_{1}=\left[g_{j}\right]_{1}+C_{i, j, k}\left[g_{j}\right]_{k} \text { for } 1 \leq i \leq n+1 \text { with } i \neq j
$$

Note that (39) is true irrespective of what system we are in.

We combine (36) and (39) to obtain

$$
\left[g_{i}\right]_{1}(t)-\left[g_{j}\right]_{1}(t)=C_{i, j, k}\left[g_{j}\right]_{k}\left(t_{1}\right) e^{\lambda_{h, k-1}\left(t-t_{1}\right)} \text { for } i \neq j
$$

whenever we are in system (35). Hence

$$
d_{i, j}(t)=\left|C_{i, j, k}\right|\left|\left[g_{j}\right]_{k}\left(t_{1}\right)\right| e^{\lambda_{h, k-1}\left(t-t_{1}\right)}, \text { for } t_{1} \leq t \leq t_{2} \text { and } i \neq j
$$

Consequently $\frac{d d_{i, j}(t)}{d t}<0$, or else $\left[g_{i}\right]_{1}$ and $\left[g_{j}\right]_{1}$ both agree over the time interval $\left[t_{1}, t_{2}\right]$. Thus the distance between $\left[g_{i}\right]_{1}(t)$ and the constant $\left[g_{j}\right]_{1}(t)$ is either getting smaller or always zero over the time interval $\left[t_{1}, t_{2}\right]$, when we are in the system described by $\dot{\bar{x}}=\bar{A}_{j, h} \bar{x}$.

\section{Proof of Theorem 3.1 :}

We will now prove that $\lim _{t \rightarrow \infty}\left(x_{1}, x_{2}, \ldots, x_{n}\right)=0$, for any solution $\left(x_{1}, x_{2}, \ldots, x_{n}\right)$ to the system (1) with the set $\mathcal{A}$ defined as in statement of Theorem 3.1, and then we will be done. Denote the maximum value (minimum value) of $G(t)$, for a time $t$ in the time interval $\left[t_{1}, t_{2}\right]$, by $\max ^{1} G(t)\left(\min ^{1} G(t)\right)$. Recall that we are in system $(35)$ when $t \in\left[t_{1}, t_{2}\right]$. Then

$$
\begin{aligned}
\max ^{1} G(t)-\min ^{1} G(t) & =\left[g_{i}\right]_{1}(t)-\left[g_{r}\right]_{1}(t), \text { for some } i, r \in\{1,2, \ldots, n+1\} \\
& =\left[g_{i}\right]_{1}(t)-\left[g_{j}\right]_{1}(t)+\left[g_{j}\right]_{1}(t)-\left[g_{r}\right]_{1}(t) \\
& =\left|C_{i, j, k}\right|\left|\left[g_{j}\right]_{k}\left(t_{1}\right)\right| e^{\lambda_{h, k-1}\left(t-t_{1}\right)}+\left|C_{r, j, q}\right|\left|\left[g_{j}\right]_{q}\left(t_{1}\right)\right| e^{\lambda_{h, q-1}\left(t-t_{1}\right)}
\end{aligned}
$$

where, as in Lemma $3.2, k=j$, if $i=1$, and $k=i$, if $i \neq 1$. Similarly $q=j$, if $r=1$, and $q=r$, if $r \neq 1$. Note that if $\left[g_{j}\right]_{1}$ is a maximum value (or minimum value) of $G(t)$, then the last line above collapses to just one term instead of two, and in this case the following arguments will also work. Now let $B_{i, j, t_{1}}=\left|C_{i, j, k}\right|\left|\left[g_{j}\right]_{k}\left(t_{1}\right)\right|=$ distance between $\left[g_{i}\right]_{1}\left(t_{1}\right)$ and $\left[g_{j}\right]_{1}\left(t_{1}\right)$. Let $B_{r, j, t_{1}}=\left|C_{r, j, q}\right|\left|\left[g_{j}\right]_{q}\left(t_{1}\right)\right|=$ distance between $\left[g_{j}\right]_{1}\left(t_{1}\right)$ and $\left[g_{r}\right]_{1}\left(t_{1}\right)$. Also let 
$\lambda=\max \left\{\lambda_{\alpha, \beta}: 1 \leq \alpha \leq p, 1 \leq \beta \leq n\right\}$. Note that $\lambda<0$. Then

$$
\begin{aligned}
\max ^{1} G(t)-\min ^{1} G(t) & \leq\left(B_{i, j, t_{1}}+B_{r, j, t_{1}}\right) e^{\lambda\left(t-t_{1}\right)} \\
& \leq\left(\max ^{1} G\left(t_{1}\right)-\min ^{1} G\left(t_{1}\right)\right) e^{\lambda\left(t-t_{1}\right)}
\end{aligned}
$$

The last inequality follows from the fact that, over the time interval $\left[t_{1}, t_{2}\right],\left[g_{i}\right]_{1}(t)$ remains on the same side of the constant $\left[g_{j}\right]_{1}(t)$ and $\left[g_{r}\right]_{1}(t)$ remains on the other side of $\left[g_{j}\right]_{1}(t)$. This is because the right hand side of (40) does not change sign as time changes over the time interval $\left[t_{1}, t_{2}\right]$. Note that $i$ and $r$ may change with time and so $\max ^{1} G\left(t_{1}\right)$ may not correspond to $\left[g_{i}\right]_{1}\left(t_{1}\right)$, and $\min ^{1} G\left(t_{1}\right)$ may not correspond to $\left[g_{r}\right]_{1}\left(t_{1}\right)$.

Now suppose we switch to the next (second) system described by

$$
\dot{\bar{x}}=\bar{A}_{c, w} \bar{x}
$$

over the time interval $\left[t_{2}, t_{3}\right]$. Denote the maximum value (minimum value) of $G(t)$, for some time $t \in\left[t_{2}, t_{3}\right]$, by $\max ^{2} G(t)\left(\min ^{2} G(t)\right)$. Then as above we get

$$
\begin{aligned}
\max ^{2} G(t)-\min ^{2} G(t) & \leq\left(\max ^{2} G\left(t_{2}\right)-\min ^{2} G\left(t_{2}\right)\right) e^{\lambda\left(t-t_{2}\right)} \\
& \leq\left(\max ^{1} G\left(t_{1}\right)-\min ^{1} G\left(t_{1}\right)\right) e^{\lambda\left(t_{2}-t_{1}\right)} e^{\lambda\left(t-t_{2}\right)} \\
& \leq\left(\max ^{1} G\left(t_{1}\right)-\min ^{1} G\left(t_{1}\right)\right) e^{\lambda\left(t-t_{1}\right)}
\end{aligned}
$$

The second inequality above follows from the fact that we start the second system (42) at time $t_{2}$ when we stop the first system (35), and the initial conditions for the second system are the terminal conditions for the first system at time $t_{2}$. Thus $\max ^{2} G\left(t_{2}\right)-\min ^{2} G\left(t_{2}\right) \leq$ $\left(\max ^{1} G\left(t_{1}\right)-\min ^{1} G\left(t_{1}\right)\right) e^{\lambda\left(t_{2}-t_{1}\right)}$ from $(41)$.

Now suppose we switch to the next (third) system described by

$$
\dot{\bar{x}}=\bar{A}_{d, u} \bar{x}
$$

over the time interval $\left[t_{3}, t_{4}\right]$. Denote the maximum value (minimum value) of $G(t)$, for some time $t \in\left[t_{3}, t_{4}\right]$, by $\max ^{3} G(t)\left(\min ^{3} G(t)\right)$. Then as above we get

$$
\max ^{3} G(t)-\min ^{3} G(t) \leq\left(\max ^{1} G\left(t_{1}\right)-\min ^{1} G\left(t_{1}\right)\right) e^{\lambda\left(t-t_{1}\right)}
$$


For the general suituation, when we have switched for the $m^{\text {th }}$ time, we are in the system described by $\dot{\bar{x}}=\bar{A}_{z, l} \bar{x}$ over the time interval $\left[t_{m}, t_{m+1}\right]$. Again we denote the maximum value (minimum value) of $G(t)$, for some time $t \in\left[t_{m}, t_{m+1}\right]$, by $\max ^{m} G(t)\left(\min ^{m} G(t)\right)$. Then as above we get

$$
\max ^{m} G(t)-\min ^{m} G(t) \leq\left(\max ^{1} G\left(t_{1}\right)-\min ^{1} G\left(t_{1}\right)\right) e^{\lambda\left(t-t_{1}\right)}
$$

Therefore, since $\lambda<0$, we have $\lim _{t \rightarrow \infty}(\max G(t)-\min G(t))=0$, where $\max G(t)(\min G(t))$ denotes the maximum value (minimum value) of $G(t)$ for any time $t \geq t_{1}$. Thus

$$
\begin{aligned}
\lim _{t \rightarrow \infty}\left|\left[g_{i}\right]_{1}(t)-\left[g_{j}\right]_{1}(t)\right| & =0, \text { for all } i, j \in\{1,2, \ldots, n+1\} . \\
\lim _{t \rightarrow \infty}\left|C_{i, j, k}\right|\left|\left[g_{j}\right]_{k}(t)\right| & =0, \text { where } k=j \text { if } i=1, \text { and } k=i \text {, if } i \neq 1, \text { and } i \neq j . \\
\lim _{t \rightarrow \infty}\left|\left[g_{j}\right]_{k}(t)\right| & =0, \text { for } j \in\{1,2, \ldots, n+1\}, \text { and } k \in\{2,3, \ldots, n+1\} .
\end{aligned}
$$

The second line above follows from (39), which is independent of what system we are in. The last line above follows from the fact that the $C_{i, j, k}^{\prime s}$ form a finite collection of non-zero numbers when $i \neq j$. Also note that the last line above might not hold for $k=1$ because then $i=j=1$. Therefore, since $\lim _{t \rightarrow \infty} \bar{x}(t)=\lim _{t \rightarrow \infty} \bar{M}_{j} g_{j}(t)$, we get

$$
\begin{aligned}
& \lim _{t \rightarrow \infty}\left(\begin{array}{c}
x_{n+1} \\
x_{1} \\
x_{2} \\
\vdots \\
x_{n}
\end{array}\right)=\lim _{t \rightarrow \infty}\left(\begin{array}{cccccc}
1 & b & 1 & 1 & \ldots & \ldots \\
0 & & & & \\
0 & & M_{j} & & \\
0 & & & & \\
\vdots & & & & \\
0 & & & &
\end{array}\right)\left(\begin{array}{c}
{\left[g_{j}\right]_{1}(t)} \\
{\left[g_{j}\right]_{2}(t)} \\
\vdots \\
{\left[g_{j}\right]_{n+1}(t)}
\end{array}\right) \\
& \lim _{t \rightarrow \infty}\left(\begin{array}{c}
x_{1} \\
x_{2} \\
\vdots \\
x_{n}
\end{array}\right)=\lim _{t \rightarrow \infty}\left(\begin{array}{cc}
0 & \\
0 & M_{j} \\
\vdots & \\
0 &
\end{array}\right)\left(\begin{array}{c}
{\left[g_{j}\right]_{1}(t)} \\
{\left[g_{j}\right]_{2}(t)} \\
\vdots \\
{\left[g_{j}\right]_{n+1}(t)}
\end{array}\right)
\end{aligned}
$$




$$
=\left(\begin{array}{c}
0 \\
0 \\
\vdots \\
0
\end{array}\right)
$$

Thus

$$
\lim _{t \rightarrow \infty}\left(x_{1}, x_{2}, \ldots, x_{n}\right)=0
$$

and we have global attractivity of the origin in the switching system (1). Q.E.D. 


\section{References}

Angeli, D. (1999), A note on the stability of arbitrarily switched homogeneous systems, Submitted to systems and control letters.

Kato, T. (1980), Perturbation theory for linear operators, Springer.

Liberzon, D., Hespanha, J. P. \& Morse, S. (1998), Stability of Switched Linear Systems: A Lie Algebraic Condition, Technical report, Laboratory for Control Science and Engineering, Yale University.

Mohler, R. (1991), Nonlinear Systems: Dynamics and Control, Prentice Hall.

Mori, Y., Mori, T. \& Kuroe, Y. (1996), Classes of Systems having Common Quadratic Lyapunov Functions: Triangular System Matrices, in 'proceedings of Electronic Information and Systems Conference (in Japanese)'.

Mori, Y., Mori, T. \& Kuroe, Y. (1997), A Solution to the Common Lyapunov Function Problem for Continuous Time Systems, in "proceedings of 36th Conference on Decision and Control'.

Narendra, K. \& Annaswamy, A. (1989), Stable Adaptive Systems, Prentice-Hall.

Narendra, K. S. \& Balakrishnan, J. (1994), 'A Common Lyapunov Function for Stable LTI Systems with Commuting $\mathcal{A}$-Matrices', IEEE Transactions on Automatic Control $\mathbf{3 9}(12), 2469-2471$.

Power, H. \& Simpson, R. (1978), An Introduction to Dynamics and Control, McGraw-Hill.

Rugh, W. (1996), Linear system theory, Prentice Hall.

Shorten, R. (1996), A Study of Hybrid Dynamical Systems with Application to Automotive Control, PhD thesis, Department of Electrical and Electronic Engineering, University College Dublin, Republic of Ireland. 
Shorten, R. \& Narendra, K. (1997), A Sufficient Condition for the Existence of a Common Lyapunov Function for Two Second Order Systems: Part 1., Technical report, Center for Systems Science, Yale University.

Shorten, R. \& Narendra, K. (1998), On the Stability and Existence of Common Lyapunov Functions for Linear Stable Switching Systems, In proceedings of Conference on Decision and Control, Florida Dec 15th-18th.

Shorten, R. \& Ó Cairbre, F. (n.d.), A Proof Of Global Attractivity for a Class of Switching Systems using a non-Lyapunov Approach, Accepted for publication by Institute of Mathematics and its Applications : Journal of Mathematical Control and Information.

Shorten, R. N. \& Ó Cairbre, F. (2000), On the global attractivity of a class of switching systems, in 'Proceedings of the American Control Conference'.

Shorten, R. N., Ó Cairbre, F. \& Curran, P. F. (2000), On the dynamic instability of a class of switching systems, in 'Proceedings of IFAC conference on Artificial Intelligence in Real Time Control'. 